\title{
LINEAR QUADRATIC STOCHASTIC TWO-PERSON ZERO-SUM DIFFERENTIAL GAMES IN AN INFINITE HORIZON *
}

\author{
Jingrui Sun ${ }^{1}$, Jiongmin Yong ${ }^{2}$ And Shuguang Zhang ${ }^{3}$
}

\begin{abstract}
This paper is concerned with a linear quadratic stochastic two-person zero-sum differential game with constant coefficients in an infinite time horizon. Open-loop and closed-loop saddle points are introduced. The existence of closed-loop saddle points is characterized by the solvability of an algebraic Riccati equation with a certain stabilizing condition. A crucial result makes our approach work is the unique solvability of a class of linear backward stochastic differential equations in an infinite horizon.
\end{abstract}

Mathematics Subject Classification. 93E20, 91A23, 49N10, 49N70.

Received April 28, 2014. Revised March 28, 2015.

Published online May 16, 2016.

\section{INTRODUCTION}

Let $(\Omega, \mathcal{F}, \mathbb{F}, \mathbb{P})$ be a complete filtered probability space on which a one-dimensional standard Brownian motion $W(\cdot)$ is defined with $\mathbb{F}=\left\{\mathcal{F}_{t}\right\}_{t \geq 0}$ being its natural filtration augmented by all the $\mathbb{P}$-null sets in $\mathcal{F}[11,19]$. Consider the following controlled linear stochastic differential equation (SDE, for short) on the infinite time horizon $[0, \infty)$ :

$$
\left\{\begin{array}{l}
\mathrm{d} X(t)=\left[A X(t)+B_{1} u_{1}(t)+B_{2} u_{2}(t)+b(t)\right] \mathrm{d} t \\
\quad+\left[C X(t)+D_{1} u_{1}(t)+D_{2} u_{2}(t)+\sigma(t)\right] \mathrm{d} W(t), \quad t \geq 0, \\
X(0)=x,
\end{array}\right.
$$

where $A, C \in \mathbb{R}^{n \times n}$ and $B_{i} \in \mathbb{R}^{n \times m_{i}}(i=1,2)$ are given (deterministic) matrices; $b(\cdot)$ and $\sigma(\cdot)$ are $\mathbb{R}^{n}$-valued, $\mathbb{F}$-progressively measurable, square integrable processes. In the above, $X(\cdot)$, valued in $\mathbb{R}^{n}$, is called the state process with initial state $x \in \mathbb{R}^{n} ;$ for $i=1,2, u_{i}(\cdot)$, valued in $\mathbb{R}^{m_{i}}$, is called the control process of Player $i$. Let $\mathbb{H}$

Keywords and phrases. Linear quadratic stochastic differential game, two-person, zero-sum, infinite horizon, open-loop and closed-loop saddle points, algebraic Riccati equation, stabilizing solution.

* This work is supported in part by NSF Grants DMS-1007514 and DMS-1406776, China Scholarship Council, NSFC Grants 11471304 and 11401556, and the fundamental research funds for the central universities (No. WK2040000012).

1 School of Mathematical Sciences, University of Science and Technology of China, Hefei, Anhui 230026, P.R. of China. sjr@mail.ustc.edu.cn

2 Department of Mathematics, University of Central Florida, Orlando, FL 32816, USA. jiongmin.yong@ucf.edu

3 Department of Statistics and Finance, University of Science and Technology of China, Hefei, Anhui 230026, P.R. China.

sgzhang@ustc.edu.cn 
be a Euclidean space and $T>0$, we introduce the following:

$$
\begin{aligned}
& L_{\mathbb{F}}^{2}(\mathbb{H})=\left\{\varphi:[0, \infty) \times \Omega \rightarrow \mathbb{H} \mid \varphi(\cdot) \text { is } \mathbb{F} \text {-progressively measurable, } \mathbb{E} \int_{0}^{\infty}|\varphi(t)|^{2} \mathrm{~d} t<\infty\right\}, \\
& \mathcal{X}[0, T]=\left\{X:[0, \infty) \times \Omega \rightarrow \mathbb{R}^{n} \mid X(\cdot) \text { is } \mathbb{F} \text {-adapted, continuous, } \mathbb{E}\left(\sup _{0 \leq t \leq T}|X(t)|^{2}\right)<\infty\right\}, \\
& \mathcal{X}_{l o c}[0, \infty)=\bigcap_{T>0} \mathcal{X}[0, T], \quad \mathcal{X}[0, \infty)=\left\{\left.X(\cdot) \in \mathcal{X}_{l o c}[0, \infty)\left|\mathbb{E} \int_{0}^{\infty}\right| X(t)\right|^{2} \mathrm{~d} t<\infty\right\} .
\end{aligned}
$$

By a standard argument using contraction mapping theorem, one can show that for any initial state $x \in \mathbb{R}^{n}$ and control pair $\left(u_{1}(\cdot), u_{2}(\cdot)\right) \in L_{\mathbb{F}}^{2}\left(\mathbb{R}^{m_{1}}\right) \times L_{\mathbb{F}}^{2}\left(\mathbb{R}^{m_{2}}\right)$, state equation (1.1) admits a unique solution $X(\cdot) \equiv$ $X\left(\cdot ; x, u_{1}(\cdot), u_{2}(\cdot)\right) \in \mathcal{X}_{l o c}[0, \infty)$. Next, we introduce the following performance functional:

$$
\begin{aligned}
& J\left(x ; u_{1}(\cdot), u_{2}(\cdot)\right) \\
& \triangleq \mathbb{E} \int_{0}^{\infty}\left[\left\langle\left(\begin{array}{ccc}
Q & S_{1}^{T} & S_{2}^{T} \\
S_{1} & R_{11} & R_{12} \\
S_{2} & R_{21} & R_{22}
\end{array}\right)\left(\begin{array}{l}
X(t) \\
u_{1}(t) \\
u_{2}(t)
\end{array}\right),\left(\begin{array}{l}
X(t) \\
u_{1}(t) \\
u_{2}(t)
\end{array}\right)\right\rangle+2\left\langle\left(\begin{array}{c}
q(t) \\
\rho_{1}(t) \\
\rho_{2}(t)
\end{array}\right),\left(\begin{array}{l}
X(t) \\
u_{1}(t) \\
u_{2}(t)
\end{array}\right)\right\rangle\right] \mathrm{d} t,
\end{aligned}
$$

where

$$
Q \in \mathbb{S}^{n}, S_{i} \in \mathbb{R}^{m_{i} \times n}, R_{i i} \in \mathbb{S}^{m_{i}}, R_{21}^{T}=R_{12} \in \mathbb{R}^{m_{1} \times m_{2}}, q(\cdot) \in L_{\mathbb{F}}^{2}\left(\mathbb{R}^{n}\right), \rho_{i}(\cdot) \in L_{\mathbb{F}}^{2}\left(\mathbb{R}^{m_{i}}\right) ; \quad i=1,2 .
$$

In the above, $\mathbb{S}^{k}$ is the set of all $(k \times k)$ symmetric matrices, and $M^{T}$ is the transpose of $M$ (a matrix or a vector); $X(\cdot)=X\left(\cdot ; x, u_{1}(\cdot), u_{2}(\cdot)\right)$ on the right hand side of $(1.2)$ is the corresponding state process. Note that in general, for $\left(x, u_{1}(\cdot), u_{2}(\cdot)\right) \in \mathbb{R}^{n} \times L_{\mathbb{F}}^{2}\left(\mathbb{R}^{m_{1}}\right) \times L_{\mathbb{F}}^{2}\left(\mathbb{R}^{m_{2}}\right)$, the solution $X(\cdot) \equiv X\left(\cdot ; x, u_{1}(\cdot), u_{2}(\cdot)\right)$ of $(1.1)$ might just be in $\mathcal{X}_{l o c}[0, \infty)$ and the above performance functional $J\left(x ; u_{1}(\cdot), u_{2}(\cdot)\right)$ might not be defined. Therefore, we introduce the following set:

$$
\mathcal{U}_{a d}(x) \triangleq\left\{\left(u_{1}(\cdot), u_{2}(\cdot)\right) \in L_{\mathbb{F}}^{2}\left(\mathbb{R}^{m_{1}}\right) \times L_{\mathbb{F}}^{2}\left(\mathbb{R}^{m_{2}}\right) \mid X\left(\cdot ; x, u_{1}(\cdot), u_{2}(\cdot)\right) \in \mathcal{X}[0, \infty)\right\}, \quad x \in \mathbb{R}^{n} .
$$

Any element $\left(u_{1}(\cdot), u_{2}(\cdot)\right) \in \mathcal{U}_{a d}(x)$ is called an admissible control pair for the initial state $x$ and the corresponding $X(\cdot)=X\left(\cdot ; x, u_{1}(\cdot), u_{2}(\cdot)\right)$ is called an admissible state process with the initial state $x$. Roughly speaking, in the game, Player 1 wishes to minimize $(1.2)$ by selecting a control $u_{1}(\cdot)$, and Player 2 wishes to maximize (1.2) by selecting a control $u_{2}(\cdot)$. Therefore, (1.2) represents the cost for Player 1 and the payoff for Player 2 . The problem is to find an admissible control pair $\left(u_{1}^{*}(\cdot), u_{2}^{*}(\cdot)\right)$ that both players can accept, and we refer to such a problem as a linear quadratic (LQ, for short) stochastic two-person zero-sum differential game, denoted by Problem (LQG). There are basically two types of controls for both players: open-loop controls and closed-loop controls. An open-loop control usually depends on the initial state as well as all the information, including those of the opponent, over the whole time duration $[0, \infty)$, whereas a closed-loop control is required to be independent of the initial state, and the future information. Thus, in reality, it is more meaningful and convenient to using closed-loop controls rather than open-loop controls. However, mathematically, open-loop controls are still meaningful and they are actually helpful in finding "optimal" closed-loop controls.

Let us briefly recall some relevant history. In 1965, deterministic LQ two-person zero-sum differential games in finite horizon (LQDG problem, for short) were introduced and studied by Ho et al. [8]. In 1970, Schmitendorf [15] studied both open-loop and closed-loop strategies for LQDG problems. Among other things, it was shown that the existence of a closed-loop saddle point may not imply that of an open-loop saddle point. In 1979, Bernhard [5] carefully investigated LQDG problems from closed-loop point of view; see also the book by Basar and Bernhard [3] in this aspect. In 2005, Zhang [20] proved that for an LQDG problem, the existence of the open-loop value is equivalent to the finiteness of the corresponding open-loop lower and upper values, which is 
also equivalent to the existence of an open-loop saddle point. Along this line, there were a couple of follow-up works [6,7] appeared afterwards. In 2006, Mou and Yong [12] studied a stochastic LQ two-person zero-sum differential game in finite horizon from an open-loop point of view, by means of Hilbert space method. On the other hand, in 1976, Ichikawa [10] studied a deterministic LQ two-person zero-sum differential game on $[0, \infty)$ in a Hilbert space and deduced some sufficient conditions for the existence of closed-loop saddle points. In 2000, Ait Rami et al. [1] studied an LQ stochastic optimal control problem on $[0, \infty)$, followed by the work of Wu and Zhou [17]. Recently, based on the work of Yong [18], Huang et al. [9] studied a mean-field LQ optimal control problem on $[0, \infty)$.

The rest of the paper is organized as follows. In Section 2, we collect some preliminary results. Section 3 is devoted to the unique solvability of a linear backward stochastic differential equation (BSDE, for short) on $[0, \infty)$. In Section 4, we discuss closed-loop optimal controls of Problem (LQ) and deduce a necessary condition for the existence of a closed-loop optimal control via the solvability of an algebraic Riccati equation (ARE, for short). In Section 5, we pose our differential game problem and characterize closed-loop saddle points by means of algebraic Riccati equations. Some examples are presented in Section 6.

\section{Preliminary Results}

Let us begin by considering a stochastic optimal control problem. The state equation takes the following form:

$$
\left\{\begin{array}{l}
\mathrm{d} X(t)=[A X(t)+B u(t)+b(t)] \mathrm{d} t+[C X(t)+D u(t)+\sigma(t)] \mathrm{d} W(t), \quad t \geq 0, \\
X(0)=x
\end{array}\right.
$$

with cost functional

$$
J(x ; u(\cdot))=\mathbb{E} \int_{0}^{\infty}\left[\left\langle\left(\begin{array}{cc}
Q & S^{T} \\
S & R
\end{array}\right)\left(\begin{array}{c}
X(t) \\
u(t)
\end{array}\right),\left(\begin{array}{c}
X(t) \\
u(t)
\end{array}\right)\right\rangle+2\left\langle\left(\begin{array}{c}
q(t) \\
\rho(t)
\end{array}\right),\left(\begin{array}{c}
X(t) \\
u(t)
\end{array}\right)\right\rangle\right] \mathrm{d} t,
$$

where $A, C \in \mathbb{R}^{n \times n}, B, D \in \mathbb{R}^{n \times m}, Q \in \mathbb{S}^{n}, R \in \mathbb{S}^{m}, S \in \mathbb{R}^{m \times n}$, and $b(\cdot), \sigma(\cdot), q(\cdot) \in L_{\mathbb{F}}^{2}\left(\mathbb{R}^{n}\right), \rho(\cdot) \in L_{\mathbb{F}}^{2}\left(\mathbb{R}^{m}\right)$. The solution of $(2.1)$ is denoted by $X(\cdot ; x, u(\cdot))$. For any given $x \in \mathbb{R}^{n}$, the set of admissible controls is defined by the following:

$$
\mathcal{U}_{a d}(x) \triangleq\left\{u(\cdot) \in L_{\mathbb{F}}^{2}\left(\mathbb{R}^{m}\right) \mid X(\cdot ; x, u(\cdot)) \in \mathcal{X}[0, \infty)\right\} .
$$

Clearly, $\mathcal{U}_{a d}(x)$ is a convex subset of $L_{\mathbb{F}}^{2}\left(\mathbb{R}^{m}\right)$, but not necessarily a subspace of $L_{\mathbb{F}}^{2}\left(\mathbb{R}^{m}\right)$ in general. We pose the following problem.

Problem (LQ). For given $x \in \mathbb{R}^{n}$, find a $\bar{u}(\cdot) \in \mathcal{U}_{a d}(x)$, such that

$$
V(x) \triangleq J(x ; \bar{u}(\cdot))=\inf _{u(\cdot) \in \mathcal{U}_{a d}(x)} J(x ; u(\cdot))
$$

Any $\bar{u}(\cdot) \in \mathcal{U}_{a d}(x)$ satisfying (2.3) is called an open-loop optimal control of Problem (LQ), and the corresponding $\bar{X}(\cdot) \equiv X(\cdot ; x, \bar{u}(\cdot))$ is called an optimal state process. The function $V(\cdot)$ is called the value function of Problem (LQ). The following notions are similar to those introduced in [19].

\section{Definition 2.1 .}

(i) Problem (LQ) is said to be finite if

$$
V(x)>-\infty, \quad \forall x \in \mathbb{R}^{n} .
$$

(ii) Problem (LQ) is said to be (uniquely) solvable if it has a (unique) open-loop optimal control. 
When $b(\cdot)=\sigma(\cdot)=0$, we briefly denote the system (2.1) by $[A, C ; B, D]$. We also denote by $[A, C]$ the following uncontrolled system:

$$
\left\{\begin{array}{l}
\mathrm{d} X(t)=A X(t) \mathrm{d} t+C X(t) \mathrm{d} W(t), \quad t \geq 0 \\
X(0)=x
\end{array}\right.
$$

When $b(\cdot), \sigma(\cdot), q(\cdot), \rho(\cdot)=0$, we denote the corresponding Problem (LQ) by Problem (LQ) ${ }^{0}$. The corresponding cost functional and value function are denoted by $J^{0}(x ; u(\cdot))$ and $V^{0}(x)$, respectively.

We note that, in general, the admissible control set $\mathcal{U}_{a d}(x)$ may be empty for some $x \in \mathbb{R}^{n}$. To avoid such a case, we introduce the following definition.

\section{Definition 2.2.}

(i) System $[A, C]$ is said to be $L^{2}$-exponentially stable if for any $x \in \mathbb{R}^{n}$, the solution $X(\cdot) \equiv X(\cdot ; x) \in$ $\mathcal{X}_{l o c}[0, \infty)$ of $(2.5)$ satisfies the following:

$$
\lim _{t \rightarrow \infty} \mathrm{e}^{\lambda t} \mathbb{E}|X(t)|^{2}=0, \quad \text { for some } \lambda>0 .
$$

(ii) System $[A, C]$ is said to be $L^{2}$-globally integrable if for any $x \in \mathbb{R}^{n}$, the solution $X(\cdot) \equiv X(\cdot ; x) \in \mathcal{X}_{\text {loc }}[0, \infty)$ of $(2.5)$ is in $\mathcal{X}[0, \infty)$.

(iii) System $[A, C]$ is said to be $L^{2}$-asymptotically stable if for any $x \in \mathbb{R}^{n}$, the solution $X(\cdot) \equiv X(\cdot ; x) \in$ $\mathcal{X}_{l o c}[0, \infty)$ of $(2.5)$ satisfies the following:

$$
\lim _{t \rightarrow \infty} \mathbb{E}|X(t)|^{2}=0
$$

The following result will be used frequently in this paper. For a proof, see [9].

Lemma 2.3. The following are equivalent:

(i) System $[A, C]$ is $L^{2}$-exponentially stable;

(ii) System $[A, C]$ is $L^{2}$-globally integrable;

(iii) For any $\Lambda>0$, the following Lyapunov equation admits a solution $P>0$ :

$$
P A+A^{T} P+C^{T} P C+\Lambda=0
$$

(iv) There exists a $P>0$ such that $P A+A^{T} P+C^{T} P C<0$;

(v) System $[A, C]$ is $L^{2}$-asymptotically stable, and there exists a $P \in \mathbb{S}^{n}$ such that

$$
P A+A^{T} P+C^{T} P C<0 .
$$

In this case, we simply say that the system $[A, C]$ is $L^{2}$-stable.

Next, we present a result concerning the $L^{2}$-integrability of the solution to the following system:

$$
\left\{\begin{array}{l}
\mathrm{d} X(t)=[A X(t)+b(t)] \mathrm{d} t+[C X(t)+\sigma(t)] \mathrm{d} W(t), \quad t \geq 0 \\
X(0)=x
\end{array}\right.
$$

Proposition 2.4. Let $A, C \in \mathbb{R}^{n \times n}$ and $b(\cdot), \sigma(\cdot) \in L_{\mathbb{F}}^{2}\left(\mathbb{R}^{n}\right)$. Let $X(\cdot) \equiv X(\cdot ; x)$ be the solution to the $\operatorname{SDE}(2.7)$. If $[A, C]$ is $L^{2}$-stable, then $X(\cdot) \in \mathcal{X}[0, \infty)$. 
Proof. Since $[A, C]$ is $L^{2}$-stable, by Lemma 2.3 , there exists a $P>0$ such that

$$
P A+A^{T} P+C^{T} P C \equiv-\Lambda<0 .
$$

Applying Itô's formula to $s \mapsto\langle P X(s), X(s)\rangle$, one has

$$
\begin{aligned}
& \mathbb{E}\langle P X(t), X(t)\rangle-\langle P x, x\rangle \\
&=\mathbb{E} \int_{0}^{t}\left[\left\langle\left(P A+A^{T} P+C^{T} P C\right) X(s), X(s)\right\rangle\right. \\
&\left.+2\left\langle P b(s)+C^{T} P \sigma(s), X(s)\right\rangle+\langle P \sigma(s), \sigma(s)\rangle\right] \mathrm{d} s \\
&= \mathbb{E} \int_{0}^{t}\left[-\langle\Lambda X(s), X(s)\rangle+2\left\langle P b(s)+C^{T} P \sigma(s), X(s)\right\rangle+\langle P \sigma(s), \sigma(s)\rangle\right] \mathrm{d} s, \quad \forall t \geq 0 .
\end{aligned}
$$

Therefore

$$
\begin{aligned}
& \frac{\mathrm{d}}{\mathrm{d} t} \mathbb{E}\left\langle P^{\frac{1}{2}} X(t), P^{\frac{1}{2}} X(t)\right\rangle=\frac{\mathrm{d}}{\mathrm{d} t} \mathbb{E}\langle P X(t), X(t)\rangle \\
& =-\mathbb{E}\langle\Lambda X(t), X(t)\rangle+2 \mathbb{E}\left\langle P b(t)+C^{T} P \sigma(t), X(t)\right\rangle+\mathbb{E}\langle P \sigma(t), \sigma(t)\rangle \\
& =-\mathbb{E}\left\langle\Gamma P^{\frac{1}{2}} X(t), P^{\frac{1}{2}} X(t)\right\rangle+2 \mathbb{E}\left\langle\eta(t), P^{\frac{1}{2}} X(t)\right\rangle+\mathbb{E}\langle P \sigma(t), \sigma(t)\rangle,
\end{aligned}
$$

where

$$
\Gamma \triangleq P^{-\frac{1}{2}} \Lambda P^{-\frac{1}{2}}>0, \quad \eta(\cdot)=P^{\frac{1}{2}} b(\cdot)+P^{-\frac{1}{2}} C^{T} P \sigma(\cdot) .
$$

Let $\lambda>0$ be the smallest eigenvalue of $\Gamma$. By Cauchy-Schwarz's inequality, we have

$$
\begin{aligned}
& \frac{\mathrm{d}}{\mathrm{d} t} \mathbb{E}\left\langle P^{\frac{1}{2}} X(t), P^{\frac{1}{2}} X(t)\right\rangle \\
& \leq-\lambda \mathbb{E}\left\langle P^{\frac{1}{2}} X(t), P^{\frac{1}{2}} X(t)\right\rangle+\frac{\lambda}{2} \mathbb{E}\left\langle P^{\frac{1}{2}} X(t), P^{\frac{1}{2}} X(t)\right\rangle+\frac{2}{\lambda} \mathbb{E}|\eta(t)|^{2}+\mathbb{E}\langle P \sigma(t), \sigma(t)\rangle \\
& =-\frac{\lambda}{2} \mathbb{E}\left\langle P^{\frac{1}{2}} X(t), P^{\frac{1}{2}} X(t)\right\rangle+\frac{2}{\lambda} \mathbb{E}|\eta(t)|^{2}+\mathbb{E}\langle P \sigma(t), \sigma(t)\rangle .
\end{aligned}
$$

Let $\mu>0$ be the smallest eigenvalue of $P$. By Gronwall's inequality, we obtain

$$
\begin{aligned}
\mu \mathbb{E}|X(t)|^{2} & \leq \mathbb{E}\left\langle P^{\frac{1}{2}} X(t), P^{\frac{1}{2}} X(t)\right\rangle \\
& \leq \mathrm{e}^{-\frac{\lambda}{2} t}\langle P x, x\rangle+\int_{0}^{t} \mathrm{e}^{-\frac{\lambda}{2}(t-s)}\left[\frac{2}{\lambda} \mathbb{E}|\eta(s)|^{2}+\mathbb{E}\langle P \sigma(s), \sigma(s)\rangle\right] \mathrm{d} s,
\end{aligned}
$$

which, together with Young's inequality, implies that $\mathbb{E}|X(\cdot)|^{2}$ is integrable over $[0, \infty)$.

Definition 2.5. System $[A, C ; B, D]$ is said to be $L^{2}$-stabilizable if there exists a $\Theta \in \mathbb{R}^{m \times n}$ such that $[A+$ $B \Theta, C+D \Theta]$ is $L^{2}$-stable. In this case, $\Theta$ is called a stabilizer of $[A, C ; B, D]$. We denote the set of all stabilizers of $[A, C ; B, D]$ by $\mathscr{S} \equiv \mathscr{S}[A, C ; B, D]$.

We now introduce the following assumption.

(H1) System $[A, C ; B, D]$ is $L^{2}$-stabilizable, i.e.,

$$
\mathscr{S}[A, C ; B, D] \neq \varnothing .
$$

By Proposition 2.4, we see that under $(\mathrm{H} 1), \mathcal{U}_{a d}(x)$ is nonempty for any $x \in \mathbb{R}^{n}$. Moreover, we have the following proposition. 
Proposition 2.6. Let (H1) hold. Then for any $x \in \mathbb{R}^{n}, u(\cdot) \in \mathcal{U}_{a d}(x)$ if and only if

$$
u(\cdot)=\Theta X(\cdot)+v(\cdot),
$$

for some $\Theta \in \mathscr{S}[A, C ; B, D]$ and $v(\cdot) \in L_{\mathbb{F}}^{2}\left(\mathbb{R}^{m}\right)$, where $X(\cdot)$ is the solution of the following SDE:

$$
\left\{\begin{array}{l}
\mathrm{d} X(t)=[(A+B \Theta) X(t)+B v(t)+b(t)] \mathrm{d} t \\
\quad+[(C+D \Theta) X(t)+D v(t)+\sigma(t)] \mathrm{d} W(t), \quad t \geq 0 \\
X(0)=x
\end{array}\right.
$$

Proof. Let $v(\cdot) \in L_{\mathbb{F}}^{2}\left(\mathbb{R}^{m}\right)$ and $X(\cdot)$ be the solution of $(2.10)$. Since $[A+B \Theta, C+D \Theta]$ is $L^{2}$-stable, by Proposition $2.4, X(\cdot) \in \mathcal{X}[0, \infty)$. Set

$$
u(\cdot) \triangleq \Theta X(\cdot)+v(\cdot) \in L_{\mathbb{F}}^{2}\left(\mathbb{R}^{m}\right) .
$$

By uniqueness, $X(\cdot)$ also solves the following SDE:

$$
\left\{\begin{array}{l}
\mathrm{d} X(t)=[A X(t)+B u(t)+b(t)] \mathrm{d} t+[C X(t)+D u(t)+\sigma(t)] \mathrm{d} W(t), \quad t \geq 0, \\
X(0)=x
\end{array}\right.
$$

Thus, $u(\cdot) \in \mathcal{U}_{a d}(x)$.

On the other hand, suppose $u(\cdot) \in \mathcal{U}_{a d}(x)$. Let $X(\cdot) \in \mathcal{X}[0, \infty)$ be the solution of (2.11). Pick any $\Theta \in$ $\mathscr{S}[A, C ; B, D]$ and set

$$
v(\cdot) \triangleq u(\cdot)-\Theta X(\cdot) \in L_{\mathbb{F}}^{2}\left(\mathbb{R}^{m}\right) .
$$

By uniqueness, $X(\cdot)$ also solves $(2.10)$. Thus, $u(\cdot)=\Theta X(\cdot)+v(\cdot)$ with $X(\cdot)$ being the solution of $(2.10)$.

Now, we introduce the following notations:

$$
\mathscr{M}(P)=P A+A^{T} P+C^{T} P C+Q, \quad \mathscr{L}(P)=P B+C^{T} P D+S^{T}, \quad \mathscr{N}(P)=R+D^{T} P D, \quad \forall P \in \mathbb{S}^{n},
$$

and define the following convex set:

$$
\mathscr{P} \triangleq\left\{P \in \mathbb{S}^{n} \mid\left(\begin{array}{cc}
\mathscr{M}(P) & \mathscr{L}(P) \\
\mathscr{L}(P)^{T} & \mathscr{N}(P)
\end{array}\right) \geq 0\right\}
$$

The following result, found in [1], characterizes the finiteness of Problem (LQ) ${ }^{0}$.

Lemma 2.7. Problem $(\mathrm{LQ})^{0}$ is finite if and only if $\mathscr{P} \neq \varnothing$. In this case, $\mathscr{P}$ has a maximal element $P \in \mathscr{P}$ (i.e., $P \geq \widetilde{P}$, for all $\widetilde{P} \in \mathscr{P}$ ). Moreover, we have

$$
V^{0}(x)=\langle P x, x\rangle, \quad \forall x \in \mathbb{R}^{n} .
$$

\section{LiNEAR BSDES IN AN INFINITE HORIZON}

In this section, we consider the following BSDE in the infinite time horizon $[0, \infty)$ :

$$
\mathrm{d} Y(t)=-\left[A^{T} Y(t)+C^{T} Z(t)+\varphi(t)\right] \mathrm{d} t+Z(t) \mathrm{d} W(t), \quad t \in[0, \infty) .
$$

Definition 3.1. An $L^{2}$-stable adapted solution of $(3.1)$ is a pair $(Y(\cdot), Z(\cdot)) \in \mathcal{X}[0, \infty) \times L_{\mathbb{F}}^{2}\left(\mathbb{R}^{n}\right)$ satisfying

$$
Y(t)=Y(0)-\int_{0}^{t}\left[A^{T} Y(s)+C^{T} Z(s)+\varphi(s)\right] \mathrm{d} s+\int_{0}^{t} Z(s) \mathrm{d} W(s), \quad \forall t \in[0, \infty), \quad \text { a.s. }
$$


Note that by $(3.2)$, for any $T \in[0, \infty)$,

$$
Y(t)=Y(T)+\int_{t}^{T}\left[A^{T} Y(s)+C^{T} Z(s)+\varphi(s)\right] \mathrm{d} s-\int_{t}^{T} Z(s) \mathrm{d} W(s), \quad t \in[0, T], \quad \text { a.s. }
$$

Hence, letting $T \rightarrow \infty$, we have

$$
Y(t)=\int_{t}^{\infty}\left[A^{T} Y(s)+C^{T} Z(s)+\varphi(s)\right] \mathrm{d} s-\int_{t}^{\infty} Z(s) \mathrm{d} W(s), \quad t \in[0, \infty), \quad \text { a.s. }
$$

This is a familiar form of linear BSDE on $[0, \infty)$. In 2000, Peng and Shi considered the following BSDE:

$$
\mathrm{d} Y(t)=-[G(t, Y(t), Z(t))+\varphi(t)] \mathrm{d} t+Z(t) \mathrm{d} W(t), \quad t \in[0, \infty),
$$

and it was shown that, under some mild conditions, equation (3.5) admits a unique adapted solution $(Y(\cdot), Z(\cdot))$ (see [13], Thm. 4). In terms of $L^{2}$-stable adapted solutions of (3.1), we can restate the result of [13] as follows.

Proposition 3.2. Suppose

$$
A+A^{T}+C^{T} C<0 .
$$

Then for any $\varphi(\cdot) \in L_{\mathbb{F}}^{2}\left(\mathbb{R}^{n}\right)$, BSDE (3.1) admits a unique $L^{2}$-stable adapted solution $(Y(\cdot), Z(\cdot))$.

Instead of the above, we have the following result which gives the unique solvability of BSDE (3.5) under a weaker condition.

Theorem 3.3. Suppose that $[A, C]$ is $L^{2}$-stable. Then for any $\varphi(\cdot) \in L_{\mathbb{F}}^{2}\left(\mathbb{R}^{n}\right)$, BSDE (3.1) admits a unique $L^{2}$-stable adapted solution $(Y(\cdot), Z(\cdot))$.

Before proving the above result, let us make an observation. By Lemma 2.3, part (iv), taking $P=I$, we see that condition (3.6) implies the $L^{2}$-stability of $[A, C]$. On the other hand, let

$$
A=\left(\begin{array}{ll}
-1 & 1 \\
-1 & 0
\end{array}\right), \quad C=\left(\begin{array}{cc}
\frac{\sqrt{2}}{2} & 0 \\
0 & \frac{\sqrt{2}}{2}
\end{array}\right), \quad P=\left(\begin{array}{cc}
2 & -1 \\
-1 & 2
\end{array}\right)>0 .
$$

One has

$$
P A+A^{T} P+C^{T} P C=\left(\begin{array}{cc}
-1 & \frac{1}{2} \\
\frac{1}{2} & -1
\end{array}\right)<0 .
$$

By Lemma 2.3, part (iv), $[A, C]$ is $L^{2}$-stable. However,

$$
A+A^{T}+C^{T} C=\left(\begin{array}{cc}
-\frac{3}{2} & 0 \\
0 & \frac{1}{2}
\end{array}\right)
$$

which is indefinite. Thus, (3.6) fails. Hence, the condition assumed in Theorem 3.3 is weaker than that assumed in Proposition 3.2. In order to prove Theorem 3.3, we need the following a priori estimates.

Proposition 3.4. Suppose that $[A, C]$ is $L^{2}$-stable and $\varphi(\cdot) \in L_{\mathbb{F}}^{2}\left(\mathbb{R}^{n}\right)$. Let $(Y(\cdot), Z(\cdot))$ be an $L^{2}$-stable adapted solution of BSDE (3.1). Then

$$
\mathbb{E}\left(\sup _{0 \leq t<\infty}|Y(t)|^{2}\right)+\mathbb{E} \int_{0}^{\infty}|Z(t)|^{2} \mathrm{~d} t \leq K \mathbb{E} \int_{0}^{\infty}|\varphi(t)|^{2} \mathrm{~d} t
$$

Hereafter, $K>0$ represents a generic constant which can be different from line to line. 
Proof. Since $[A, C]$ is $L^{2}$-stable, by Lemma 2.3 , there exists a $P>0$ such that $P A+A^{T} P+C^{T} P C<0$. Hence, one can choose $\varepsilon>0$ such that

$$
P A+A^{T} P+(1+\varepsilon) C^{T} P C \equiv-\Lambda_{\varepsilon}<0 .
$$

Applying Itô's formula to $s \mapsto\left\langle P^{-1} Y(s), Y(s)\right\rangle$, one has that for any $0 \leq t<T<\infty$ (suppressing $s$ in the functions),

$$
\begin{aligned}
\left\langle P^{-1} Y(T), Y(T)\right\rangle-\left\langle P^{-1} Y(t), Y(t)\right\rangle \\
=-\int_{t}^{T}\left\{2\left\langle P^{-1}\left(A^{T} Y+C^{T} Z+\varphi\right), Y\right\rangle-\left\langle P^{-1} Z, Z\right\rangle\right\} \mathrm{d} s+2 \int_{t}^{T}\left\langle Z, P^{-1} Y\right\rangle \mathrm{d} W(s) \\
=-\int_{t}^{T}\left\{\left\langle P A P^{-1} Y, P^{-1} Y\right\rangle+\left\langle A^{T} P P^{-1} Y, P^{-1} Y\right\rangle+2\left\langle C^{T} Z, P^{-1} Y\right\rangle\right. \\
\left.+2\left\langle\varphi, P^{-1} Y\right\rangle-\left\langle P^{-1} Z, Z\right\rangle\right\} \mathrm{d} s+2 \int_{t}^{T}\left\langle Z, P^{-1} Y\right\rangle \mathrm{d} W(s) \\
=-\int_{t}^{T}\left\{\left\langle\left(P A+A^{T} P\right) P^{-1} Y, P^{-1} Y\right\rangle+2\left\langle\varphi, P^{-1} Y\right\rangle\right. \\
\left.+2\left\langle Z, C P^{-1} Y\right\rangle-\left\langle P^{-1} Z, Z\right\rangle\right\} \mathrm{d} s+2 \int_{t}^{T}\left\langle Z, P^{-1} Y\right\rangle \mathrm{d} W(s) \\
=-\int_{t}^{T}\left\{\left\langle-\Lambda_{\varepsilon} P^{-1} Y, P^{-1} Y\right\rangle+2\left\langle\varphi, P^{-1} Y\right\rangle-(1+\varepsilon)\left\langle P C P^{-1} Y, C P^{-1} Y\right\rangle\right. \\
\left.+2\left\langle Z, C P^{-1} Y\right\rangle-\left\langle P^{-1} Z, Z\right\rangle\right\} \mathrm{d} s+2 \int_{t}^{T}\left\langle Z, P^{-1} Y\right\rangle \mathrm{d} W(s) \\
=-\int_{t}^{T}\left\{\left\langle-\Lambda_{\varepsilon} P^{-1} Y, P^{-1} Y\right\rangle+2\left\langle\varphi, P^{-1} Y\right\rangle\right. \\
-(1+\varepsilon)\left\langle P\left[C P^{-1} Y-\frac{1}{1+\varepsilon} P^{-1} Z\right], C P^{-1} Y-\frac{1}{1+\varepsilon} P^{-1} Z\right\rangle \\
\left.-\frac{\varepsilon}{1+\varepsilon}\left\langle P^{-1} Z, Z\right\rangle\right\} \mathrm{d} s+2 \int_{t}^{T}\left\langle Z, P^{-1} Y\right\rangle \mathrm{d} W(s) .
\end{aligned}
$$

Let $\lambda>0$ be the smallest eigenvalue of $\Lambda_{\varepsilon}>0$. By Cauchy-Schwarz's inequality, we have

$$
\begin{aligned}
\langle & \left.P^{-1} Y(t), Y(t)\right\rangle-\left\langle P^{-1} Y(T), Y(T)\right\rangle+\int_{t}^{T} \frac{\varepsilon}{1+\varepsilon}\left\langle P^{-1} Z(s), Z(s)\right\rangle \mathrm{d} s \\
= & \int_{t}^{T}\left\{\left\langle-\Lambda_{\varepsilon} P^{-1} Y, P^{-1} Y\right\rangle+2\left\langle\varphi, P^{-1} Y\right\rangle-(1+\varepsilon)\left|P^{\frac{1}{2}}\left[C P^{-1} Y-\frac{1}{1+\varepsilon} P^{-1} Z\right]\right|^{2}\right\} \mathrm{d} s \\
& -2 \int_{t}^{T}\left\langle Z, P^{-1} Y\right\rangle \mathrm{d} W(s) \\
\leq & \int_{t}^{T}\left\{-\lambda\left|P^{-1} Y(s)\right|^{2}+\lambda\left|P^{-1} Y(s)\right|^{2}+\frac{1}{\lambda}|\varphi(s)|^{2}\right\} \mathrm{d} s-2 \int_{t}^{T}\left\langle Z(s), P^{-1} Y(s)\right\rangle \mathrm{d} W(s) \\
= & \frac{1}{\lambda} \int_{t}^{T}|\varphi(s)|^{2} \mathrm{~d} s-2 \int_{t}^{T}\left\langle Z(s), P^{-1} Y(s)\right\rangle \mathrm{d} W(s) .
\end{aligned}
$$


Since $Y(\cdot) \in \mathcal{X}[0, \infty)$, we must have $\lim _{T \rightarrow \infty} \mathbb{E}|Y(T)|^{2}=0$. Taking expectation on both sides of (3.8), and letting $T \rightarrow \infty$, one has (noting that $P>0$ )

$$
\mathbb{E}|Y(t)|^{2}+\mathbb{E} \int_{t}^{\infty}|Z(s)|^{2} \mathrm{~d} s \leq K \mathbb{E} \int_{t}^{\infty}|\varphi(s)|^{2} \mathrm{~d} s, \quad \forall t \in[0, \infty)
$$

On the other hand, by Burkholder-Davis-Gundy's inequality, we have (noting (3.9))

$$
\begin{aligned}
& \mathbb{E}\left\{\sup _{0 \leq t \leq T}\left|\int_{t}^{T}\left\langle Z(s), P^{-1} Y(s)\right\rangle \mathrm{d} W(s)\right|\right\} \leq 2 \mathbb{E}\left\{\sup _{0 \leq t \leq T}\left|\int_{0}^{t}\left\langle Z(s), P^{-1} Y(s)\right\rangle \mathrm{d} W(s)\right|\right\} \\
& \leq K \mathbb{E}\left\{\int_{0}^{T}\left|\left\langle Z(s), P^{-1} Y(s)\right\rangle\right|^{2} \mathrm{~d} s\right\}^{\frac{1}{2}} \leq K \mathbb{E}\left\{\int_{0}^{T}\left|P^{-\frac{1}{2}} Z(s)\right|^{2}\left|P^{-\frac{1}{2}} Y(s)\right|^{2} \mathrm{~d} s\right\}^{\frac{1}{2}} \\
& \leq K \mathbb{E}\left\{\left(\sup _{0 \leq t \leq T}\left|P^{-\frac{1}{2}} Y(t)\right|^{2}\right)^{\frac{1}{2}}\left(\int_{0}^{T}\left|P^{-\frac{1}{2}} Z(s)\right|^{2} \mathrm{~d} s\right)^{\frac{1}{2}}\right\} \\
& \leq \frac{1}{4} \mathbb{E}\left(\sup _{0 \leq t \leq T}\left|P^{-\frac{1}{2}} Y(t)\right|^{2}\right)+K \mathbb{E} \int_{0}^{T}|Z(s)|^{2} \mathrm{~d} s \\
& \leq \frac{1}{4} \mathbb{E}\left(\sup _{0 \leq t \leq T}\left|P^{-\frac{1}{2}} Y(t)\right|^{2}\right)+K \mathbb{E} \int_{0}^{\infty}|\varphi(s)|^{2} \mathrm{~d} s .
\end{aligned}
$$

Consequently, from (3.8), we obtain (using (3.9) and (3.10))

$$
\begin{aligned}
& \mathbb{E}\left(\sup _{0 \leq t \leq T}\left|P^{-\frac{1}{2}} Y(t)\right|^{2}\right)=\mathbb{E}\left(\sup _{0 \leq t \leq T}\left\langle P^{-1} Y(t), Y(t)\right\rangle\right) \\
& \leq \mathbb{E}\left\langle P^{-1} Y(T), Y(T)\right\rangle+\frac{1}{\lambda} \mathbb{E} \int_{0}^{T}|\varphi(s)|^{2} \mathrm{~d} s+2 \mathbb{E}\left\{\sup _{0 \leq t \leq T}\left|\int_{t}^{T}\left\langle Z(s), P^{-1} Y(s)\right\rangle \mathrm{d} W(s)\right|\right\} \\
& \leq K \mathbb{E} \int_{0}^{\infty}|\varphi(s)|^{2} \mathrm{~d} s+2 \mathbb{E}\left\{\sup _{0 \leq t \leq T}\left|\int_{t}^{T}\left\langle Z(s), P^{-1} Y(s)\right\rangle \mathrm{d} W(s)\right|\right\} \\
& \leq \frac{1}{4} \mathbb{E}\left(\sup _{0 \leq t \leq T}\left|P^{-\frac{1}{2}} Y(t)\right|^{2}\right)+K \mathbb{E} \int_{0}^{\infty}|\varphi(s)|^{2} \mathrm{~d} s .
\end{aligned}
$$

Therefore (noting $P>0$ again),

$$
\mathbb{E}\left(\sup _{0 \leq t \leq T}|Y(t)|^{2}\right) \leq K \mathbb{E} \int_{0}^{\infty}|\varphi(s)|^{2} \mathrm{~d} s, \quad \forall T \in[0, \infty)
$$

Combining (3.9) and (3.11), making use of Fatou's Lemma, yields (3.7).

Proposition 3.5. Under the hypotheses of Proposition 3.4, we have

$$
\mathbb{E} \int_{0}^{\infty}|Y(t)|^{2} \mathrm{~d} t \leq K \mathbb{E} \int_{0}^{\infty}|\varphi(t)|^{2} \mathrm{~d} t
$$


Proof. Let $P>0$ be the matrix in the proof of Proposition 3.4. Applying Itô's formula to $s \mapsto\left\langle P^{-1} Y(s), Y(s)\right\rangle$, one has that for any $0 \leq t<\infty$,

$$
\begin{aligned}
& \mathbb{E}\left\langle P^{-1} Y(t), Y(t)\right\rangle-\mathbb{E}\left\langle P^{-1} Y(0), Y(0)\right\rangle \\
& =\mathbb{E} \int_{0}^{t}\left\{-\left\langle P^{-1}\left[A^{T} Y+C^{T} Z+\varphi\right], Y\right\rangle-\left\langle P^{-1} Y, A^{T} Y+C^{T} Z+\varphi\right\rangle+\left\langle P^{-1} Z, Z\right\rangle\right\} \mathrm{d} s \\
& =\mathbb{E} \int_{0}^{t}\left\{-\left\langle P A P^{-1} Y, P^{-1} Y\right\rangle-\left\langle A^{T} P P^{-1} Y, P^{-1} Y\right\rangle-2\left\langle C^{T} Z+\varphi, P^{-1} Y\right\rangle+\left\langle P^{-1} Z, Z\right\rangle\right\} \mathrm{d} s \\
& \geq \mathbb{E} \int_{0}^{t}\left\{-\left\langle\left[P A+A^{T} P\right] P^{-1} Y, P^{-1} Y\right\rangle-2\left\langle C^{T} Z+\varphi, P^{-1} Y\right\rangle\right\} \mathrm{d} s .
\end{aligned}
$$

Let $\mu>0$ be the smallest eigenvalue of $-\left(P A+A^{T} P\right)>0$. By Cauchy-Schwarz's inequality, we have

$$
\begin{aligned}
& \mathbb{E}\left\langle P^{-1} Y(t), Y(t)\right\rangle-\mathbb{E}\left\langle P^{-1} Y(0), Y(0)\right\rangle \\
& \geq \mathbb{E} \int_{0}^{t}\left\{\mu\left|P^{-1} Y(s)\right|^{2}-\frac{\mu}{2}\left|P^{-1} Y(s)\right|^{2}-\frac{4}{\mu}\left|C^{T} Z(s)\right|^{2}-\frac{4}{\mu}|\varphi(s)|^{2}\right\} \mathrm{d} s, \quad \forall t \in[0, \infty) .
\end{aligned}
$$

Letting $t \rightarrow \infty$ in (3.13), one has

$$
\mathbb{E}\left\langle P^{-1} Y(0), Y(0)\right\rangle+\frac{\mu}{2} \mathbb{E} \int_{0}^{\infty}\left|P^{-1} Y(s)\right|^{2} \mathrm{~d} s \leq \frac{4}{\mu} \mathbb{E} \int_{0}^{\infty}\left(\left|C^{T} Z(s)\right|^{2}+|\varphi(s)|^{2}\right) \mathrm{d} s .
$$

Combining the a priori estimate (3.7) we obtain the desired estimate (3.12).

Proof of Theorem 3.3. The uniqueness is an immediate consequence of the a priori estimate (3.7). We now prove the existence. For $k=1,2, \ldots$, we set

$$
\varphi_{k}(t) \triangleq 1_{[0, k]}(t) \varphi(t), \quad t \in[0, \infty) .
$$

Clearly, $\left\{\varphi_{k}(\cdot)\right\}_{k=1}^{\infty}$ converges to $\varphi(\cdot)$ in $L_{\mathbb{F}}^{2}\left(\mathbb{R}^{n}\right)$.

We now consider, for each $k$, the $L^{2}$-stable adapted solution $\left(Y_{k}(\cdot), Z_{k}(\cdot)\right)$ of the following BSDE:

$$
\mathrm{d} Y_{k}(t)=-\left[A^{T} Y_{k}(t)+C^{T} Z_{k}(t)+\varphi_{k}(t)\right] \mathrm{d} t+Z_{k}(t) \mathrm{d} W(t), \quad t \in[0, \infty) .
$$

The above can be solved as follows: on $[0, k],\left(Y_{k}(\cdot), Z_{k}(\cdot)\right)$ is the adapted solution to the following BSDE:

$$
\left\{\begin{array}{l}
\mathrm{d} Y_{k}(t)=-\left[A^{T} Y_{k}(t)+C^{T} Z_{k}(t)+\varphi_{k}(t)\right] \mathrm{d} t+Z_{k}(t) \mathrm{d} W(t), \quad t \in[0, k], \\
Y_{k}(k)=0,
\end{array}\right.
$$

and on $(k, \infty)$, it is identically equal to zero. By Proposition 3.4 and 3.5 , we have

$$
\begin{aligned}
& \mathbb{E}\left(\sup _{0 \leq t<\infty}\left|Y_{k}(t)-Y_{j}(t)\right|^{2}\right)+\mathbb{E} \int_{0}^{\infty}\left|Y_{k}(t)-Y_{j}(t)\right|^{2} \mathrm{~d} t+\mathbb{E} \int_{0}^{\infty}\left|Z_{k}(t)-Z_{j}(t)\right|^{2} \mathrm{~d} t \\
& \leq K \mathbb{E} \int_{0}^{\infty}\left|\varphi_{k}(t)-\varphi_{j}(t)\right|^{2} \mathrm{~d} t, \quad \forall k, j .
\end{aligned}
$$

Therefore, there exists a $(Y(\cdot), Z(\cdot)) \in \mathcal{X}[0, \infty) \times L_{\mathbb{F}}^{2}\left(\mathbb{R}^{n}\right)$ such that

$$
\mathbb{E}\left(\sup _{0 \leq t<\infty}\left|Y_{k}(t)-Y(t)\right|^{2}\right)+\mathbb{E} \int_{0}^{\infty}\left|Z_{k}(t)-Z(t)\right|^{2} \mathrm{~d} t \rightarrow 0, \quad \text { as } \quad k \rightarrow \infty,
$$

which implies that $(Y(\cdot), Z(\cdot))$ is an $L^{2}$-stable adapted solution of (3.1). 


\section{Closed-loop optimal Controls}

In this section we discuss the closed-loop optimal controls of Problem (LQ). Let us first recall that for any $M \in \mathbb{R}^{m \times n}$, there exists a unique matrix $M^{\dagger} \in \mathbb{R}^{n \times m}$, called the (Moore-Penrose) pseudo-inverse of $M$, satisfying the following ([14]):

$$
M M^{\dagger} M=M, \quad M^{\dagger} M M^{\dagger}=M^{\dagger}, \quad\left(M M^{\dagger}\right)^{T}=M M^{\dagger}, \quad\left(M^{\dagger} M\right)^{T}=M^{\dagger} M .
$$

In addition, if $M \in \mathbb{S}^{n}$, then $M^{\dagger} \in \mathbb{S}^{n}$, and

$$
M M^{\dagger}=M^{\dagger} M ; \quad M \geq 0 \Longleftrightarrow M^{\dagger} \geq 0 .
$$

Lemma 4.1 (Extended Schur's Lemma [2]). Let $M \in \mathbb{S}^{n}, N \in \mathbb{S}^{m}, L \in \mathbb{R}^{n \times m}$. Then the following conditions are equivalent:

(i) $M-L N^{\dagger} L^{T} \geq 0, N \geq 0$, and $L\left(I-N N^{\dagger}\right)=0$.

(ii) $\left(\begin{array}{cc}M & L \\ L^{T} & N\end{array}\right) \geq 0$.

Note that $L\left(I-N N^{\dagger}\right)=0$ is equivalent to $\mathcal{R}\left(L^{T}\right) \subseteq \mathcal{R}(N)$, where $\mathcal{R}(\Lambda)$ is the range of a matrix $\Lambda$. We now introduce the following notion.

Definition 4.2. A pair $\left(\Theta^{*}, u^{*}(\cdot)\right) \in \mathscr{S} \times L_{\mathbb{F}}^{2}\left(\mathbb{R}^{m}\right)$ is called a closed-loop optimal control of Problem (LQ) if

$$
J\left(x ; \Theta^{*} X^{*}(\cdot)+u^{*}(\cdot)\right) \leq J(x ; \Theta X(\cdot)+u(\cdot)), \quad \forall(x, \Theta, u(\cdot)) \in \mathbb{R}^{n} \times \mathscr{S} \times L_{\mathbb{F}}^{2}\left(\mathbb{R}^{m}\right) .
$$

The following technical result, which is similar to Berkovitz's equivalence lemma for LQDG problems found in [4], can be shown by a simple adoption of ([16], Prop. 3.3).

Proposition 4.3. For $\left(\Theta^{*}, u^{*}(\cdot)\right) \in \mathscr{S} \times L_{\mathbb{F}}^{2}\left(\mathbb{R}^{m}\right)$, the following statements are equivalent:

(i) $\left(\Theta^{*}, u^{*}(\cdot)\right)$ is a closed-loop optimal control of Problem (LQ).

(ii) For any $x \in \mathbb{R}^{n}$ and $u(\cdot) \in L_{\mathbb{F}}^{2}\left(\mathbb{R}^{m}\right)$, the following holds:

$$
J\left(x ; \Theta^{*} X^{*}(\cdot)+u^{*}(\cdot)\right) \leq J\left(x ; \Theta^{*} X(\cdot)+u(\cdot)\right) .
$$

Now we present a characterization of closed-loop optimal controls of Problem (LQ) in terms of infinite horizon forward-backward stochastic differential equations (FBSDE, for short).

Theorem 4.4. A pair $\left(\Theta^{*}, u^{*}(\cdot)\right) \in \mathscr{S} \times L_{\mathbb{F}}^{2}\left(\mathbb{R}^{m}\right)$ is a closed-loop optimal control of Problem (LQ) if and only if for any $x \in \mathbb{R}^{n}$, the following FBSDE admits an adapted solution $\left(X^{*}(\cdot), Y^{*}(\cdot), Z^{*}(\cdot)\right) \in \mathcal{X}[0, \infty) \times \mathcal{X}[0, \infty) \times$ $L_{\mathbb{F}}^{2}\left(\mathbb{R}^{n}\right)$ :

$$
\left\{\begin{array}{l}
\mathrm{d} X^{*}(t)=\left[\left(A+B \Theta^{*}\right) X^{*}+B u^{*}+b\right] \mathrm{d} t+\left[\left(C+D \Theta^{*}\right) X^{*}+D u^{*}+\sigma\right] \mathrm{d} W(t), \quad t \geq 0, \\
\mathrm{~d} Y^{*}(t)=-\left[A^{T} Y^{*}+C^{T} Z^{*}+\left(Q+S^{T} \Theta^{*}\right) X^{*}+S^{T} u^{*}+q\right] \mathrm{d} t+Z^{*} \mathrm{~d} W(t), \quad t \geq 0, \\
X^{*}(0)=x
\end{array}\right.
$$

such that the following stationarity condition holds:

$$
R u^{*}+B^{T} Y^{*}+D^{T} Z^{*}+\left(S+R \Theta^{*}\right) X^{*}+\rho=0, \quad \text { a.e. a.s. }
$$

and

$$
\mathbb{E} \int_{0}^{\infty}\left\langle\left(\begin{array}{cc}
Q & S^{T} \\
S & R
\end{array}\right)\left(\begin{array}{c}
X_{0} \\
\Theta^{*} X_{0}+u
\end{array}\right),\left(\begin{array}{c}
X_{0} \\
\Theta^{*} X_{0}+u
\end{array}\right)\right\rangle \mathrm{d} t \geq 0, \quad \forall u(\cdot) \in L_{\mathbb{F}}^{2}\left(\mathbb{R}^{m}\right),
$$

where $X_{0}(\cdot)$ is the solution of

$$
\left\{\begin{array}{l}
\mathrm{d} X_{0}(t)=\left[\left(A+B \Theta^{*}\right) X_{0}(t)+B u(t)\right] \mathrm{d} t+\left[\left(C+D \Theta^{*}\right) X_{0}(t)+D u(t)\right] \mathrm{d} W(t), \quad t \geq 0, \\
X_{0}(0)=0
\end{array}\right.
$$


Proof. Consider the state equation

$$
\left\{\begin{aligned}
\mathrm{d} X(t)= & {\left[\left(A+B \Theta^{*}\right) X(t)+B u(t)+b(t)\right] \mathrm{d} t } \\
& +\left[\left(C+D \Theta^{*}\right) X(t)+D u(t)+\sigma(t)\right] \mathrm{d} W(t), \quad t \geq 0 \\
X(0)= & x
\end{aligned}\right.
$$

with the cost functional

$$
\begin{aligned}
& \widetilde{J}(x ; u(\cdot)) \equiv J\left(x ; \Theta^{*} X(\cdot)+u(\cdot)\right) \\
& =\mathbb{E} \int_{0}^{\infty}\left[\left\langle\left(\begin{array}{cc}
Q & S^{T} \\
S & R
\end{array}\right)\left(\begin{array}{c}
X \\
\Theta^{*} X+u
\end{array}\right),\left(\begin{array}{c}
X \\
\Theta^{*} X+u
\end{array}\right)\right\rangle+2\left\langle\left(\begin{array}{c}
q \\
\rho
\end{array}\right),\left(\begin{array}{c}
X \\
\Theta^{*} X+u
\end{array}\right)\right\rangle\right] \mathrm{d} t \\
& =\mathbb{E} \int_{0}^{\infty}\left[\left\langle\left(\begin{array}{cc}
\widetilde{Q} & \widetilde{S}^{T} \\
\widetilde{S} & R
\end{array}\right)\left(\begin{array}{c}
X \\
u
\end{array}\right),\left(\begin{array}{c}
X \\
u
\end{array}\right)\right\rangle+2\left\langle\left(\begin{array}{c}
\widetilde{q} \\
\rho
\end{array}\right),\left(\begin{array}{c}
X \\
u
\end{array}\right)\right\rangle\right] \mathrm{d} t,
\end{aligned}
$$

where

$$
\widetilde{Q}=Q+\left(\Theta^{*}\right)^{T} S+S^{T} \Theta^{*}+\left(\Theta^{*}\right)^{T} R \Theta^{*}, \quad \widetilde{S}=S+R \Theta^{*}, \quad \widetilde{q}=q+\left(\Theta^{*}\right)^{T} \rho .
$$

By Proposition 4.3, $\left(\Theta^{*}, u^{*}(\cdot)\right)$ is a closed-loop optimal control of Problem (LQ) if and only if for any $x \in \mathbb{R}^{n}$, $u^{*}(\cdot)$ is an open-loop optimal control for the problem with the above state equation and cost functional. For any $u(\cdot) \in L_{\mathbb{F}}^{2}\left(\mathbb{R}^{m}\right)$ and $\varepsilon \in \mathbb{R}$, let $X^{\varepsilon}(\cdot)$ be the solution of

$$
\left\{\begin{aligned}
\mathrm{d} X^{\varepsilon}(t)= & \left\{\left(A+B \Theta^{*}\right) X^{\varepsilon}(t)+B\left[u^{*}(t)+\varepsilon u(t)\right]+b(t)\right\} \mathrm{d} t \\
& +\left\{\left(C+D \Theta^{*}\right) X^{\varepsilon}(t)+D\left[u^{*}(t)+\varepsilon u(t)\right]+\sigma(t)\right\} \mathrm{d} W(t), \quad t \geq 0 \\
X^{\varepsilon}(0)= & x
\end{aligned}\right.
$$

Thus, $X_{0}(\cdot) \equiv \frac{X^{\varepsilon}(\cdot)-X^{*}(\cdot)}{\varepsilon}$ is independent of $\varepsilon$ and satisfies (4.6). Then

$$
\begin{aligned}
& \widetilde{J}\left(x ; u^{*}(\cdot)+\varepsilon u(\cdot)\right)-\widetilde{J}\left(x ; u^{*}(\cdot)\right) \\
&=\varepsilon \mathbb{E} \int_{0}^{\infty}\left[\left\langle\left(\begin{array}{cc}
\widetilde{Q} & \widetilde{S}^{T} \\
\widetilde{S} & R
\end{array}\right)\left(\begin{array}{c}
2 X^{*}(t)+\varepsilon X_{0}(t) \\
2 u^{*}(t)+\varepsilon u(t)
\end{array}\right),\left(\begin{array}{c}
X_{0}(t) \\
u(t)
\end{array}\right)\right\rangle+2\left\langle\left(\begin{array}{c}
\widetilde{q}(t) \\
\rho(t)
\end{array}\right),\left(\begin{array}{c}
X_{0}(t) \\
u(t)
\end{array}\right)\right\rangle\right] \mathrm{d} t \\
&= 2 \varepsilon \mathbb{E} \int_{0}^{\infty}\left[\left\langle\widetilde{Q} X^{*}, X_{0}\right\rangle+\left\langle\widetilde{S} X^{*}, u\right\rangle+\left\langle\widetilde{S} X_{0}, u^{*}\right\rangle+\left\langle R u^{*}, u\right\rangle+\left\langle\widetilde{q}, X_{0}\right\rangle+\langle\rho, u\rangle\right] \mathrm{d} t \\
&+\varepsilon^{2} \mathbb{E} \int_{0}^{\infty}\left[\left\langle\widetilde{Q} X_{0}(t), X_{0}(t)\right\rangle+2\left\langle\widetilde{S} X_{0}(t), u(t)\right\rangle+\langle R u(t), u(t)\rangle\right] \mathrm{d} t \\
&= 2 \varepsilon \mathbb{E} \int_{0}^{\infty}\left[\left\langle\widetilde{Q} X^{*}+\widetilde{S}^{T} u^{*}+\widetilde{q}, X_{0}\right\rangle+\left\langle\widetilde{S} X^{*}+R u^{*}+\rho, u\right\rangle\right] \mathrm{d} t \\
&+\varepsilon^{2} \mathbb{E} \int_{0}^{\infty}\left[\left\langle\widetilde{Q} X_{0}(t), X_{0}(t)\right\rangle+2\left\langle\widetilde{S} X_{0}(t), u(t)\right\rangle+\langle R u(t), u(t)\rangle\right] \mathrm{d} t .
\end{aligned}
$$

Since $\left[A+B \Theta^{*}, C+D \Theta^{*}\right]$ is $L^{2}$-stable, by Theorem 3.3, the following BSDE:

$$
\begin{aligned}
\mathrm{d} Y^{*}= & -\left\{\left(A+B \Theta^{*}\right)^{T} Y^{*}+\left(C+D \Theta^{*}\right)^{T} Z^{*}+\widetilde{Q} X^{*}+\widetilde{S}^{T} u^{*}+\widetilde{q}\right\} \mathrm{d} t+Z^{*} \mathrm{~d} W(t) \\
= & -\left\{A^{T} Y^{*}+C^{T} Z^{*}+Q X^{*}+S^{T}\left(\Theta^{*} X^{*}+u^{*}\right)+q\right. \\
& \left.+\left(\Theta^{*}\right)^{T}\left[B^{T} Y^{*}+D^{T} Z^{*}+\left(S+R \Theta^{*}\right) X^{*}+R u^{*}+\rho\right]\right\} \mathrm{d} t+Z^{*} \mathrm{~d} W(t), \quad t \geq 0
\end{aligned}
$$


admits a unique $L^{2}$-stable adapted solution $\left(Y^{*}(\cdot), Z^{*}(\cdot)\right)$. By Itô's formula, we have

$$
\begin{aligned}
\mathbb{E}\left\langle Y^{*}(t), X_{0}(t)\right\rangle= & \mathbb{E} \int_{0}^{t}\left[-\left\langle\left(A+B \Theta^{*}\right)^{T} Y^{*}+\left(C+D \Theta^{*}\right)^{T} Z^{*}+\widetilde{Q} X^{*}+\widetilde{S}^{T} u^{*}+\widetilde{q}\right), X_{0}\right\rangle \\
& \left.+\left\langle Y^{*},\left(A+B \Theta^{*}\right) X_{0}+B u\right\rangle+\left\langle Z^{*},\left(C+D \Theta^{*}\right) X_{0}+D u\right\rangle\right] d s \\
= & \mathbb{E} \int_{0}^{t}\left[-\left\langle\widetilde{Q} X^{*}+\widetilde{S}^{T} u^{*}+\widetilde{q}, X_{0}\right\rangle+\left\langle B^{T} Y^{*}+D^{T} Z^{*}, u\right\rangle\right] d s, \quad \forall t \geq 0 .
\end{aligned}
$$

Note that

$$
\lim _{t \rightarrow \infty}\left|\mathbb{E}\left\langle Y^{*}(t), X_{0}(t)\right\rangle\right|^{2} \leq \lim _{t \rightarrow \infty} \mathbb{E}\left|Y^{*}(t)\right|^{2} \mathbb{E}\left|X_{0}(t)\right|^{2}=0 .
$$

Letting $t \rightarrow \infty$ in (4.7), one has

$$
\mathbb{E} \int_{0}^{\infty}\left\langle\widetilde{Q} X^{*}+\widetilde{S}^{T} u^{*}+\widetilde{q}, X_{0}\right\rangle d s=\mathbb{E} \int_{0}^{\infty}\left\langle B^{T} Y^{*}+D^{T} Z^{*}, u\right\rangle d s
$$

Hence,

$$
\begin{aligned}
& \widetilde{J}\left(x ; u^{*}(\cdot)+\varepsilon u(\cdot)\right)-\widetilde{J}\left(x ; u^{*}(\cdot)\right) \\
= & 2 \varepsilon \mathbb{E} \int_{0}^{\infty}\left[\left\langle\widetilde{Q} X^{*}+\widetilde{S}^{T} u^{*}+\widetilde{q}, X_{0}\right\rangle+\left\langle\widetilde{S} X^{*}+R u^{*}+\rho, u\right\rangle\right] \mathrm{d} t \\
& +\varepsilon^{2} \mathbb{E} \int_{0}^{\infty}\left[\left\langle\widetilde{Q} X_{0}(t), X_{0}(t)\right\rangle+2\left\langle\widetilde{S} X_{0}(t), u(t)\right\rangle+\langle R u(t), u(t)\rangle\right] \mathrm{d} t \\
= & 2 \varepsilon \mathbb{E} \int_{0}^{\infty}\left\langle B^{T} Y^{*}+D^{T} Z^{*}+\widetilde{S} X^{*}+R u^{*}+\rho, u\right\rangle \mathrm{d} t \\
& +\varepsilon^{2} \mathbb{E} \int_{0}^{\infty}\left\langle\left(\begin{array}{cc}
Q & S^{T} \\
S & R
\end{array}\right)\left(\begin{array}{c}
X_{0} \\
\Theta^{*} X_{0}+u
\end{array}\right),\left(\begin{array}{c}
X_{0} \\
\Theta^{*} X_{0}+u
\end{array}\right)\right\rangle \mathrm{d} t .
\end{aligned}
$$

Therefore, $\left(\Theta^{*}, u^{*}(\cdot)\right)$ is a closed-loop optimal control of Problem (LQ) if and only if (4.4) and (4.5) hold. Consequently, $\left(Y^{*}(\cdot), Z^{*}(\cdot)\right)$ solves the following BSDE:

$$
\mathrm{d} Y^{*}=-\left[A^{T} Y^{*}+C^{T} Z^{*}+Q X^{*}+S^{T}\left(\Theta^{*} X^{*}+u^{*}\right)+q\right] \mathrm{d} t+Z^{*} \mathrm{~d} W(t), \quad t \geq 0 .
$$

This completes the proof.

As a consequence, we have the following result.

Corollary 4.5. If $\left(\Theta^{*}, u^{*}(\cdot)\right)$ is a closed-loop optimal control of Problem (LQ), then $\left(\Theta^{*}, 0\right)$ is a closed-loop optimal control of Problem (LQ) ${ }^{0}$.

Proof. Let $\left(\Theta^{*}, u^{*}(\cdot)\right)$ be a closed-loop optimal control of Problem (LQ). Then, by Theorem 4.4, (4.5) holds, and for any $x \in \mathbb{R}^{n}$, FBSDE (4.3) admits an adapted solution $\left(X^{*}(\cdot), Y^{*}(\cdot), Z^{*}(\cdot)\right) \in \mathcal{X}[0, \infty) \times \mathcal{X}[0, \infty) \times L_{\mathbb{F}}^{2}\left(\mathbb{R}^{n}\right)$ satisfying (4.4). Since FBSDE (4.3) admits a solution for each $x \in \mathbb{R}^{n}$, and $\left(\Theta^{*}, u^{*}(\cdot)\right)$ is independent of $x$, by subtracting solutions corresponding $x$ and 0 , the later from the former, we see that for any $x \in \mathbb{R}^{n}$, the following FBSDE:

$$
\left\{\begin{array}{l}
\mathrm{d} X=\left(A+B \Theta^{*}\right) X \mathrm{~d} t+\left(C+D \Theta^{*}\right) X \mathrm{~d} W(t), \quad t \geq 0, \\
\mathrm{~d} Y=-\left[A^{T} Y+C^{T} Z+\left(Q+S^{T} \Theta^{*}\right) X\right] \mathrm{d} t+Z \mathrm{~d} W(t), \quad t \geq 0, \\
X(0)=x
\end{array}\right.
$$

admits an adapted solution $(X(\cdot), Y(\cdot), Z(\cdot)) \in \mathcal{X}[0, \infty) \times \mathcal{X}[0, \infty) \times L_{\mathbb{F}}^{2}\left(\mathbb{R}^{n}\right)$ satisfying

$$
B^{T} Y+D^{T} Z+\left(S+R \Theta^{*}\right) X=0, \quad \text { a.e. a.s. }
$$

Again, by Theorem 4.4, we see that $\left(\Theta^{*}, 0\right)$ is a closed-loop optimal control of Problem $(\mathrm{LQ})^{0}$. 
The following theorem gives a necessary condition for the existence of a closed-loop optimal control of Problem (LQ).

Theorem 4.6. Suppose Problem (LQ) admits a closed-loop optimal control. Then the following ARE:

$$
P A+A^{T} P+C^{T} P C+Q-\left(P B+C^{T} P D+S^{T}\right)\left(R+D^{T} P D\right)^{\dagger}\left(B^{T} P+D^{T} P C+S\right)=0
$$

admits a solution $P \in \mathbb{S}^{n}$ such that

$$
R+D^{T} P D \geq 0, \quad \mathcal{R}\left(B^{T} P+D^{T} P C+S\right) \subseteq \mathcal{R}\left(R+D^{T} P D\right),
$$

and there exists $a \Pi \in \mathbb{R}^{m \times n}$ such that

$$
-\left(R+D^{T} P D\right)^{\dagger}\left(B^{T} P+D^{T} P C+S\right)+\left[I-\left(R+D^{T} P D\right)^{\dagger}\left(R+D^{T} P D\right)\right] \Pi
$$

is a stabilizer of $[A, C ; B, D]$.

Proof. Let $\left(\Theta^{*}, u^{*}(\cdot)\right)$ be a closed-loop optimal control of Problem (LQ). Then, by Corollary $4.5,\left(\Theta^{*}, 0\right)$ is a closed-loop optimal control of Problem (LQ) ${ }^{0}$, and hence Problem $(\mathrm{LQ})^{0}$ is finite. Lemma 2.7 yields that the set $\mathscr{P}$ has a maximal element $P \in \mathscr{P}$ such that $V^{0}(x)=\langle P x, x\rangle$, and

$$
\left(\begin{array}{ll}
\mathscr{M}(P) & \mathscr{L}(P) \\
\mathscr{L}(P)^{T} & \mathscr{N}(P)
\end{array}\right) \geq 0
$$

Applying Lemma 4.1 to (4.11), we have

$$
\begin{gathered}
\mathscr{M}(P)-\mathscr{L}(P) \mathscr{N}(P)^{\dagger} \mathscr{L}(P)^{T} \geq 0, \\
\mathscr{N}(P) \geq 0, \quad \mathscr{L}(P)\left[I-\mathscr{N}(P) \mathscr{N}(P)^{\dagger}\right]=0 .
\end{gathered}
$$

Note that (4.13) is equivalent to $(4.9)$. Let $X^{*}(\cdot)$ be the solution of

$$
\left\{\begin{array}{l}
\mathrm{d} X^{*}(t)=\left(A+B \Theta^{*}\right) X^{*}(t) \mathrm{d} t+\left(C+D \Theta^{*}\right) X^{*}(t) \mathrm{d} W(t), \quad t \geq 0 \\
X(0)=x
\end{array}\right.
$$

Applying Itô's formula to $t \rightarrow\langle P X(t), X(t)\rangle$, one has

$$
\begin{aligned}
\langle P x, x\rangle= & -\mathbb{E} \int_{0}^{\infty}\left\{\left\langle\left[P\left(A+B \Theta^{*}\right)+\left(A+B \Theta^{*}\right)^{T} P\right] X, X\right\rangle+\left\langle P\left(C+D \Theta^{*}\right) X,\left(C+D \Theta^{*}\right) X\right\rangle\right\} \mathrm{d} t \\
= & -\mathbb{E} \int_{0}^{\infty}\left\langle\left[\left(P A+A^{T} P+C^{T} P C\right)+\left(P B+C^{T} P D\right) \Theta^{*}\right.\right. \\
& \left.\left.+\left(\Theta^{*}\right)^{T}\left(B^{T} P+D^{T} P C\right)+\left(\Theta^{*}\right)^{T} D^{T} P D \Theta^{*}\right] X, X\right\rangle \mathrm{d} t \\
= & -\mathbb{E} \int_{0}^{\infty}\left\langle\left[\mathscr{M}(P)+\mathscr{L}(P) \Theta^{*}+\left(\Theta^{*}\right)^{T} \mathscr{L}(P)^{T}+\left(\Theta^{*}\right)^{T} \mathscr{N}(P) \Theta^{*}\right] X, X\right\rangle \mathrm{d} t \\
& +\mathbb{E} \int_{0}^{\infty}\left\langle\left[Q+S^{T} \Theta^{*}+\left(\Theta^{*}\right)^{T} S+\left(\Theta^{*}\right)^{T} R \Theta^{*}\right] X, X\right\rangle \mathrm{d} t .
\end{aligned}
$$

Then we have (noting (4.13))

$$
\begin{aligned}
V^{0}(x)= & J^{0}\left(x, \Theta^{*} X(\cdot)\right)=\mathbb{E} \int_{0}^{\infty}\left\langle\left[Q+S^{T} \Theta^{*}+\left(\Theta^{*}\right)^{T} S+\left(\Theta^{*}\right)^{T} R \Theta^{*}\right] X, X\right\rangle \mathrm{d} t \\
= & \langle P x, x\rangle+\mathbb{E} \int_{0}^{\infty}\left\langle\left[\mathscr{M}(P)+\mathscr{L}(P) \Theta^{*}+\left(\Theta^{*}\right)^{T} \mathscr{L}(P)^{T}+\left(\Theta^{*}\right)^{T} \mathscr{N}(P) \Theta^{*}\right] X, X\right\rangle \mathrm{d} t \\
= & \langle P x, x\rangle+\mathbb{E} \int_{0}^{\infty}\left\langle\left[\mathscr{M}(P)-\mathscr{L}(P) \mathscr{N}(P)^{\dagger} \mathscr{L}(P)^{T}\right] X, X\right\rangle \mathrm{d} t \\
& +\mathbb{E} \int_{0}^{\infty}\left\langle\mathscr{N}(P)\left[\Theta^{*}+\mathscr{N}(P)^{\dagger} \mathscr{L}(P)^{T}\right] X,\left[\Theta^{*}+\mathscr{N}(P)^{\dagger} \mathscr{L}(P)^{T}\right] X\right\rangle \mathrm{d} t .
\end{aligned}
$$


Due to the equality $V^{0}(x)=\langle P x, x\rangle$ and (4.12)-(4.14), each of the two integrands on the right-hand side of (4.14) must be zero almost everywhere. Hence, we obtain

$$
\mathscr{M}(P)-\mathscr{L}(P) \mathscr{N}(P)^{\dagger} \mathscr{L}(P)^{T}=0,
$$

that is, $P$ is a solution of (4.8), and

$$
\mathscr{N}(P)^{\frac{1}{2}}\left[\Theta^{*}+\mathscr{N}(P)^{\dagger} \mathscr{L}(P)^{T}\right]=0
$$

which, together with (4.13), gives

$$
\mathscr{N}(P) \Theta^{*}+\mathscr{L}(P)^{T}=0 .
$$

Since $\mathscr{N}(P) \mathscr{N}(P)^{\dagger}$ is an orthogonal projection, we have

$$
\Theta^{*}=-\mathscr{N}(P)^{\dagger} \mathscr{L}(P)^{T}+\left[I-\mathscr{N}(P)^{\dagger} \mathscr{N}(P)\right] \Pi \in \mathscr{S},
$$

for some $\Pi \in \mathbb{R}^{n \times m}$.

We point out that the sufficiency of the above result can also be stated and proved, which is a special case of the corresponding result for two-person zero-sum differential games (see the next section). Hence, to avoid a repeating presentation, we prefer not to give the details here.

\section{OPEN-LOOP AND CLOSED-LOOP SADDLE POINTS}

We now return to our differential games. For notational simplicity, we let $m=m_{1}+m_{2}$ and denote

$$
\begin{aligned}
& B=\left(B_{1}, B_{2}\right), \quad D=\left(D_{1}, D_{2}\right), \\
& S=\left(\begin{array}{l}
S_{1} \\
S_{2}
\end{array}\right), \quad R=\left(\begin{array}{ll}
R_{11} & R_{12} \\
R_{21} & R_{22}
\end{array}\right) \equiv\left(\begin{array}{l}
R_{1} \\
R_{2}
\end{array}\right), \quad \rho(\cdot)=\left(\begin{array}{c}
\rho_{1}(\cdot) \\
\rho_{2}(\cdot)
\end{array}\right), \quad u(\cdot)=\left(\begin{array}{c}
u_{1}(\cdot) \\
u_{2}(\cdot)
\end{array}\right) .
\end{aligned}
$$

With such notations, the state equation becomes

$$
\left\{\begin{array}{l}
\mathrm{d} X(t)=[A X(t)+B u(t)+b(t)] \mathrm{d} t+[C X(t)+D u(t)+\sigma(t)] \mathrm{d} W(t), \quad t \geq 0, \\
X(0)=x
\end{array}\right.
$$

and the performance functional becomes

$$
J\left(x ; u_{1}(\cdot), u_{2}(\cdot)\right)=J(x ; u(\cdot))=\mathbb{E} \int_{0}^{\infty}\left[\left\langle\left(\begin{array}{cc}
Q & S^{T} \\
S & R
\end{array}\right)\left(\begin{array}{c}
X(t) \\
u(t)
\end{array}\right),\left(\begin{array}{c}
X(t) \\
u(t)
\end{array}\right)\right\rangle+2\left\langle\left(\begin{array}{c}
q(t) \\
\rho(t)
\end{array}\right),\left(\begin{array}{c}
X(t) \\
u(t)
\end{array}\right)\right\rangle\right] \mathrm{d} t .
$$

Also, when $b(\cdot), \sigma(\cdot), q(\cdot), \rho(\cdot)=0$, we denote the corresponding Problem (LQG) by Problem (LQG) ${ }^{0}$ and the corresponding performance functional by $J^{0}\left(x ; u_{1}(\cdot), u_{2}(\cdot)\right)$. Similar to Problem (LQ), we will assume (H1) for the system $[A, C ; B, D]$, and we also denote

$$
\mathscr{M}(P)=P A+A^{T} P+C^{T} P C+Q, \quad \mathscr{L}(P)=P B+C^{T} P D+S^{T}, \quad \mathscr{N}(P)=R+D^{T} P D ; \quad \forall P \in \mathbb{S}^{n} .
$$

Moreover, for $\Theta_{i} \in \mathbb{R}^{m_{i} \times n}, i=1,2$, we let

$$
\begin{aligned}
& \mathscr{S}_{1}\left(\Theta_{2}\right)=\left\{\Theta_{1} \in \mathbb{R}^{m_{1} \times n} \mid\left(\Theta_{1}^{T}, \Theta_{2}^{T}\right)^{T} \text { is a stabilizer of }[A, C ; B, D]\right\}, \\
& \mathscr{S}_{2}\left(\Theta_{1}\right)=\left\{\Theta_{2} \in \mathbb{R}^{m_{2} \times n} \mid\left(\Theta_{1}^{T}, \Theta_{2}^{T}\right)^{T} \text { is a stabilizer of }[A, C ; B, D]\right\} .
\end{aligned}
$$

Note that in general, say, $\mathscr{S}_{1}\left(\Theta_{2}\right)$ is not necessarily non-empty for some $\Theta_{2} \in \mathbb{R}^{m_{2} \times n}$. However, if $\Theta \equiv$ $\left(\Theta_{1}^{T}, \Theta_{2}^{T}\right)^{T} \in \mathscr{S}[A, C ; B, D]$, then both $\mathscr{S}_{1}\left(\Theta_{2}\right)$ and $\mathscr{S}_{2}\left(\Theta_{1}\right)$ are non-empty. Also, for any $x \in \mathbb{R}^{n}$, we let $\mathcal{U}_{a d}(x)$ be the set of all $u(\cdot) \equiv\left(u_{1}(\cdot), u_{2}(\cdot)\right) \in L_{\mathbb{F}}^{2}\left(\mathbb{R}^{m}\right)$ such that the corresponding state $X(\cdot) \equiv X(\cdot ; x, u(\cdot)) \in \mathcal{X}[0, \infty)$. 
Definition 5.1. For any given $x \in \mathbb{R}^{n}$, a pair $\left(\bar{u}_{1}(\cdot), \bar{u}_{2}(\cdot)\right) \in \mathcal{U}_{a d}(x)$ is called an open-loop saddle point of Problem (LQG) if

$$
J\left(x ; \bar{u}_{1}(\cdot), u_{2}(\cdot)\right) \leq J\left(x ; \bar{u}_{1}(\cdot), \bar{u}_{2}(\cdot)\right) \leq J\left(x ; u_{1}(\cdot), \bar{u}_{2}(\cdot)\right),
$$

for any $\left(u_{1}(\cdot), u_{2}(\cdot)\right) \in L_{\mathbb{F}}^{2}\left(\mathbb{R}^{m}\right)$ such that $J\left(x ; \bar{u}_{1}(\cdot), u_{2}(\cdot)\right)$ and $J\left(x ; u_{1}(\cdot), \bar{u}_{2}(\cdot)\right)$ are defined.

Definition 5.2. A 4 -tuple $\left(\Theta_{1}^{*}, u_{1}^{*}(\cdot) ; \Theta_{2}^{*}, u_{2}^{*}(\cdot)\right) \in \mathbb{R}^{m_{1} \times n} \times L_{\mathbb{F}}^{2}\left(\mathbb{R}^{m_{1}}\right) \times \mathbb{R}^{m_{2} \times n} \times L_{\mathbb{F}}^{2}\left(\mathbb{R}^{m_{2}}\right)$ is called a closed-loop saddle point of Problem (LQG) if

(i) $\Theta^{*} \equiv\left(\left(\Theta_{1}^{*}\right)^{T},\left(\Theta_{2}^{*}\right)^{T}\right)^{T} \in \mathscr{S}[A, C ; B, D]$,

(ii) for any $x \in \mathbb{R}^{n},\left(\Theta_{1}, \Theta_{2}\right) \in \mathscr{S}_{1}\left(\Theta_{2}^{*}\right) \times \mathscr{S}_{2}\left(\Theta_{1}^{*}\right)$ and $\left(u_{1}(\cdot), u_{2}(\cdot)\right) \in L_{\mathbb{F}}^{2}\left(\mathbb{R}^{m_{1}}\right) \times L_{\mathbb{F}}^{2}\left(\mathbb{R}^{m_{2}}\right)$,

$$
\begin{aligned}
J\left(x ; \Theta_{1}^{*} X(\cdot)+u_{1}^{*}(\cdot), \Theta_{2} X(\cdot)+u_{2}(\cdot)\right) & \leq J\left(x ; \Theta_{1}^{*} X^{*}(\cdot)+u_{1}^{*}(\cdot), \Theta_{2}^{*} X^{*}(\cdot)+u_{2}^{*}(\cdot)\right) \\
& \leq J\left(x ; \Theta_{1} X(\cdot)+u_{1}(\cdot), \Theta_{2}^{*}(\cdot) X(\cdot)+u_{2}^{*}(\cdot)\right) .
\end{aligned}
$$

\section{Remark 5.3.}

(a) Although both players are non-cooperative, when choosing $\Theta_{i}(i=1,2)$, they prefer to at least work together so that $\Theta=\left(\left(\Theta_{1}\right)^{T},\left(\Theta_{2}\right)^{T}\right)^{T}$ is a stabilizer of $[A, C ; B, D]$ (and the system will not be crashed). Thus, in Definition 5.2, we only require $\Theta^{*}$ being a stabilizer of $[A, C ; B, D]$ rather than $\Theta_{i}^{*}$ being a stabilizer of $\left[A, C ; B_{i}, D_{i}\right]$.

(b) By a similar method used in [16], one can show that condition (ii) in Definition 5.2 is equivalent to the following:

(ii) for any $x \in \mathbb{R}^{n}$ and $\left(u_{1}(\cdot), u_{2}(\cdot)\right) \in L_{\mathbb{F}}^{2}\left(\mathbb{R}^{m_{1}}\right) \times L_{\mathbb{F}}^{2}\left(\mathbb{R}^{m_{2}}\right)$,

$$
\begin{aligned}
J\left(x ; \Theta_{1}^{*} X(\cdot)+u_{1}^{*}(\cdot), \Theta_{2}^{*} X(\cdot)+u_{2}(\cdot)\right) & \leq J\left(x ; \Theta_{1}^{*} X^{*}(\cdot)+u_{1}^{*}(\cdot), \Theta_{2}^{*} X^{*}(\cdot)+u_{2}^{*}(\cdot)\right) \\
& \leq J\left(x ; \Theta_{1}^{*} X(\cdot)+u_{1}(\cdot), \Theta_{2}^{*}(\cdot) X(\cdot)+u_{2}^{*}(\cdot)\right) .
\end{aligned}
$$

Let $\Theta^{*}=\left(\left(\Theta_{1}^{*}\right)^{T},\left(\Theta_{2}^{*}\right)^{T}\right)^{T} \in \mathscr{S}[A, C ; B, D]$ and $u^{*}(\cdot)=\left(u_{1}^{*}(\cdot)^{T}, u_{2}^{*}(\cdot)^{T}\right)^{T} \in L_{\mathbb{F}}^{2}\left(\mathbb{R}^{m}\right)$. We look at the following state equation:

$$
\left\{\begin{array}{l}
\mathrm{d} X(t)=\left[\left(A+B \Theta^{*}\right) X(t)+B u(t)+b(t)\right] \mathrm{d} t+\left[\left(C+D \Theta^{*}\right) X(t)+D u(t)+\sigma(t)\right] \mathrm{d} W(t), \quad t \geq 0, \\
X(0)=x
\end{array}\right.
$$

and the following performance functional:

$$
\begin{aligned}
& \widetilde{J}\left(x ; u_{1}(\cdot), u_{2}(\cdot)\right) \equiv J\left(x ; \Theta_{1}^{*} X(\cdot)+u_{1}(\cdot), \Theta_{2}^{*} X(\cdot)+u_{2}(\cdot)\right) \\
& =\mathbb{E} \int_{0}^{\infty}\left[\left\langle\left(\begin{array}{cc}
\widetilde{Q} & \widetilde{S}^{T} \\
\widetilde{S} & R
\end{array}\right)\left(\begin{array}{c}
X \\
u
\end{array}\right),\left(\begin{array}{c}
X \\
u
\end{array}\right)\right\rangle+2\left\langle\left(\begin{array}{c}
\widetilde{q} \\
\rho
\end{array}\right),\left(\begin{array}{c}
X \\
u
\end{array}\right)\right\rangle\right] \mathrm{d} t,
\end{aligned}
$$

where

$$
\widetilde{Q}=Q+\left(\Theta^{*}\right)^{T} S+S^{T} \Theta^{*}+\left(\Theta^{*}\right)^{T} R \Theta^{*}, \quad \widetilde{S}=S+R \Theta^{*}, \quad \widetilde{q}=q+\left(\Theta^{*}\right)^{T} \rho .
$$

From (ii) ${ }^{\prime}$ of Remark 5.3, we see that $\left(\Theta_{1}^{*}, u_{1}^{*}(\cdot) ; \Theta_{2}^{*}, u_{2}^{*}(\cdot)\right)$ is a closed-loop saddle point of Problem (LQG) if and only if $\left(u_{1}^{*}(\cdot), u_{2}^{*}(\cdot)\right)$ is an open-loop saddle point for the problem with the above state equation and performance functional. Applying the idea used in the proof of Theorem 4.4 (see also [16], Thm. 4.1), we see that $\left(\Theta_{1}^{*}, u_{1}^{*}(\cdot) ; \Theta_{2}^{*}, u_{2}^{*}(\cdot)\right)$ is a closed-loop saddle point of Problem (LQG) if and only if for any $x \in \mathbb{R}^{n}$, the adapted solution $\left(X^{*}(\cdot), Y^{*}(\cdot), Z^{*}(\cdot)\right) \in \mathcal{X}[0, \infty) \times \mathcal{X}[0, \infty) \times L_{\mathbb{F}}^{2}\left(\mathbb{R}^{n}\right)$ of the following FBSDE:

$$
\left\{\begin{array}{l}
\mathrm{d} X^{*}(t)=\left[\left(A+B \Theta^{*}\right) X^{*}+B u^{*}+b\right] \mathrm{d} t+\left[\left(C+D \Theta^{*}\right) X^{*}+D u^{*}+\sigma\right] \mathrm{d} W(t), \quad t \geq 0, \\
\mathrm{~d} Y^{*}(t)=-\left[\left(A+B \Theta^{*}\right)^{T} Y^{*}+\left(C+D \Theta^{*}\right)^{T} Z^{*}+\widetilde{Q} X^{*}+\widetilde{S}^{T} u^{*}+\widetilde{q}\right] \mathrm{d} t+Z^{*} \mathrm{~d} W(t), \quad t \geq 0, \\
X^{*}(0)=x
\end{array}\right.
$$


satisfies the following stationarity condition:

$$
R u^{*}+B^{T} Y^{*}+D^{T} Z^{*}+\widetilde{S} X^{*}+\rho=0, \quad \text { a.e. a.s. }
$$

and the following convexity-concavity conditions hold: For $i=1,2$,

$$
(-1)^{i-1} \mathbb{E} \int_{0}^{\infty}\left\langle\left(\begin{array}{cc}
\widetilde{Q} & \widetilde{S}_{i}^{T} \\
\widetilde{S}_{i} & R_{i i}
\end{array}\right)\left(\begin{array}{c}
X_{i} \\
u_{i}
\end{array}\right),\left(\begin{array}{c}
X_{i} \\
u_{i}
\end{array}\right)\right\rangle \mathrm{d} t \geq 0, \quad \forall u_{i}(\cdot) \in L_{\mathbb{F}}^{2}\left(\mathbb{R}^{m_{i}}\right)
$$

where $\widetilde{S}_{i}=S_{i}+R_{i} \Theta^{*}$ and $X_{i}(\cdot)$ is the solution of

$$
\left\{\begin{array}{l}
\mathrm{d} X_{i}(t)=\left[\left(A+B \Theta^{*}\right) X_{i}(t)+B_{i} u_{i}(t)\right] \mathrm{d} t+\left[\left(C+D \Theta^{*}\right) X_{i}(t)+D_{i} u_{i}(t)\right] \mathrm{d} W(t), \quad t \geq 0, \\
X_{i}(0)=0 .
\end{array}\right.
$$

Applying the method used in the proof of Corollary 4.5, we obtain the following result.

Proposition 5.4. If $\left(\Theta_{1}^{*}, u_{1}^{*}(\cdot) ; \Theta_{2}^{*}, u_{2}^{*}(\cdot)\right)$ is a closed-loop saddle point of Problem (LQG), then $\left(\Theta_{1}^{*}, 0 ; \Theta_{2}^{*}, 0\right)$ is a closed-loop saddle point of Problem (LQG) ${ }^{0}$.

Next, we consider the following algebraic Riccati equation:

$$
\left\{\begin{array}{l}
P A+A^{T} P+C^{T} P C+Q-\left(P B+C^{T} P D+S^{T}\right)\left(R+D^{T} P D\right)^{\dagger}\left(B^{T} P+D^{T} P C+S\right)=0 \\
\mathcal{R}\left(B^{T} P+D^{T} P C+S\right) \subseteq \mathcal{R}\left(R+D^{T} P D\right) \\
R_{11}+D_{1}^{T} P D_{1} \geq 0, \quad R_{22}+D_{2}^{T} P D_{2} \leq 0
\end{array}\right.
$$

Definition 5.5. A $P \in \mathbb{S}^{n}$ is called a stabilizing solution of (5.10) if $P$ is a solution to (5.10) and there exists a $\Pi \in \mathbb{R}^{m \times n}$ such that

$$
-\mathscr{N}(P)^{\dagger} \mathscr{L}(P)^{T}+\left[I-\mathscr{N}(P)^{\dagger} \mathscr{N}(P)\right] \Pi \in \mathscr{S}[A, C ; B, D] .
$$

Now we give a necessary condition for the existence of closed-loop saddle points of Problem (LQG ${ }^{0}$.

Proposition 5.6. Suppose Problem (LQG) ${ }^{0}$ admits a closed-loop saddle point. Then ARE (5.10) admits a stabilizing solution $P$.

Proof. We assume without loss of generality that $\left(\Theta_{1}^{*}, 0 ; \Theta_{2}^{*}, 0\right)$ is a closed-loop saddle point of Problem (LQG) . Set

$$
V^{0}(x) \triangleq J^{0}\left(x ; \Theta_{1}^{*} X^{*}(\cdot), \Theta_{2}^{*} X^{*}(\cdot)\right)
$$

It is easily seen that $V^{0}(\cdot)$ is a quadratic form, that is, there is a $P \in \mathbb{S}^{n}$ such that

$$
V^{0}(x)=\langle P x, x\rangle, \quad \forall x \in \mathbb{R}^{n} .
$$

Consider the state equation

$$
\left\{\begin{array}{l}
\mathrm{d} X_{1}(t)=\left[\left(A+B_{2} \Theta_{2}^{*}\right) X_{1}(t)+B_{1} u_{1}(t)\right] \mathrm{d} t+\left[\left(C+D_{2} \Theta_{2}^{*}\right) X_{1}(t)+D_{1} u_{1}(t)\right] \mathrm{d} W(t), \quad t \geq 0, \\
X_{1}(0)=x
\end{array}\right.
$$

with the cost functional

$$
\begin{aligned}
& J_{1}\left(x ; u_{1}(\cdot)\right) \equiv J^{0}\left(x ; u_{1}(\cdot), \Theta_{2}^{*} X_{1}(\cdot)\right)=\mathbb{E} \int_{0}^{\infty}\left\langle\left(\begin{array}{ccc}
Q & S_{1}^{T} & S_{2}^{T} \\
S_{1} & R_{11} & R_{12} \\
S_{2} & R_{21} & R_{22}
\end{array}\right)\left(\begin{array}{c}
X_{1} \\
u_{1} \\
\Theta_{2}^{*} X_{1}
\end{array}\right),\left(\begin{array}{c}
X_{1} \\
u_{1} \\
\Theta_{2}^{*} X_{1}
\end{array}\right)\right\rangle \mathrm{d} t \\
& =\mathbb{E} \int_{0}^{\infty}\left\{\left\langle\left[Q+\left(\Theta_{2}^{*}\right)^{T} R_{22} \Theta_{2}^{*}+\left(\Theta_{2}^{*}\right)^{T} S_{2}+S_{2}^{T} \Theta_{2}^{*}\right] X_{1}, X_{1}\right\rangle+\left\langle R_{11} u_{1}, u_{1}\right\rangle+2\left\langle\left(S_{1}+R_{12} \Theta_{2}^{*}\right) X_{1}, u_{1}\right\rangle\right\} \mathrm{d} t .
\end{aligned}
$$


Then $\left(\Theta_{1}^{*}, 0\right)$ is a closed-loop optimal control of Problem $(\mathrm{LQ})^{0}$ with the above state equation and cost functional, and the value function of the above problem is given by $\langle P x, x\rangle$. By Theorem 4.6, $P$ solves the following ARE:

$$
P \widetilde{A}_{1}+\widetilde{A}_{1}^{T} P+\widetilde{C}_{1}^{T} P \widetilde{C}_{1}+\widetilde{Q}_{1}-\left(P B_{1}+\widetilde{C}_{1}^{T} P D_{1}+\widetilde{S}_{1}^{T}\right)\left(R_{11}+D_{1}^{T} P D_{1}\right)^{\dagger}\left(B_{1}^{T} P+D_{1}^{T} P \widetilde{C}_{1}+\widetilde{S}_{1}\right)=0,
$$

and (noting (4.15))

$$
R_{11}+D_{1}^{T} P D_{1} \geq 0, \quad\left(R_{11}+D_{1}^{T} P D_{1}\right) \Theta_{1}^{*}+\left(B_{1}^{T} P+D_{1}^{T} P \widetilde{C}_{1}+\widetilde{S}_{1}\right)=0,
$$

where

$$
\widetilde{A}_{1}=A+B_{2} \Theta_{2}^{*}, \quad \widetilde{C}_{1}=C+D_{2} \Theta_{2}^{*}, \quad \widetilde{Q}_{1}=Q+\left(\Theta_{2}^{*}\right)^{T} R_{22} \Theta_{2}^{*}+\left(\Theta_{2}^{*}\right)^{T} S_{2}+S_{2}^{T} \Theta_{2}^{*}, \quad \widetilde{S}_{1}=S_{1}+R_{12} \Theta_{2}^{*} .
$$

Similarly, by considering the state equation

$$
\left\{\begin{array}{l}
\mathrm{d} X_{2}(t)=\left[\left(A+B_{1} \Theta_{1}^{*}\right) X_{2}(t)+B_{2} u_{2}(t)\right] \mathrm{d} t+\left[\left(C+D_{1} \Theta_{1}^{*}\right) X_{2}(t)+D_{2} u_{2}(t)\right] \mathrm{d} W(t), \quad t \geq 0, \\
X_{2}(0)=x,
\end{array}\right.
$$

with the cost functional $J_{2}\left(x ; u_{2}(\cdot)\right) \equiv-J^{0}\left(x ; \Theta_{1}^{*} X_{2}(\cdot), u_{2}(\cdot)\right)$, we have

$$
R_{22}+D_{2}^{T} P D_{2} \leq 0, \quad\left(R_{22}+D_{2}^{T} P D_{2}\right) \Theta_{2}^{*}+\left(B_{2}^{T} P+D_{2}^{T} P \widetilde{C}_{2}+\widetilde{S}_{2}\right)=0
$$

where

$$
\widetilde{C}_{2}=C+D_{1} \Theta_{1}^{*}, \quad \widetilde{S}_{2}=S_{2}+R_{21} \Theta_{1}^{*} .
$$

Let $\Theta^{*}=\left(\left(\Theta_{1}^{*}\right)^{T},\left(\Theta_{2}^{*}\right)^{T}\right)^{T}$. Combining (5.12) and (5.13), one has

$$
\left(R+D^{T} P D\right) \Theta^{*}+\left(B^{T} P+D^{T} P C+S\right)=0,
$$

which implies

$$
\mathcal{R}\left(B^{T} P+D^{T} P C+S\right) \subseteq \mathcal{R}\left(R+D^{T} P D\right) .
$$

Since $\mathscr{N}(P)^{\dagger} \mathscr{N}(P)$ is an orthogonal projection, there exists a $\Pi \in \mathbb{R}^{m \times n}$ such that

$$
\Theta^{*}=-\mathscr{N}(P)^{\dagger} \mathscr{L}(P)^{T}+\left[I-\mathscr{N}(P)^{\dagger} \mathscr{N}(P)\right] \Pi \in \mathscr{S}[A, C ; B, D] .
$$

Using (5.11)-(5.14), we have

$$
\begin{aligned}
0= & P \widetilde{A}_{1}+\widetilde{A}_{1}^{T} P+\widetilde{C}_{1}^{T} P \widetilde{C}_{1}+\widetilde{Q}_{1}-\left(P B_{1}+\widetilde{C}_{1}^{T} P D_{1}+\widetilde{S}_{1}^{T}\right)\left(R_{11}+D_{1}^{T} P D_{1}\right)^{\dagger}\left(B_{1}^{T} P+D_{1}^{T} P \widetilde{C}_{1}+\widetilde{S}_{1}\right) \\
= & P \widetilde{A}_{1}+\widetilde{A}_{1}^{T} P+\widetilde{C}_{1}^{T} P \widetilde{C}_{1}+\widetilde{Q}_{1}-\left(\Theta_{1}^{*}\right)^{T}\left(R_{11}+D_{1}^{T} P D_{1}\right) \Theta_{1}^{*} \\
= & P A+A^{T} P+C^{T} P C+Q+\left(\Theta_{2}^{*}\right)^{T}\left(R_{22}+D_{2}^{T} P D_{2}\right) \Theta_{2}^{*}-\left(\Theta_{1}^{*}\right)^{T}\left(R_{11}+D_{1}^{T} P D_{1}\right) \Theta_{1}^{*} \\
& +\left(P B_{2}+C^{T} P D_{2}+S_{2}^{T}\right) \Theta_{2}^{*}+\left(\Theta_{2}^{*}\right)^{T}\left(B_{2}^{T} P+D_{2}^{T} P C+S_{2}\right) \\
= & P A+A^{T} P+C^{T} P C+Q-\left(\Theta_{1}^{*}\right)^{T}\left(R_{11}+D_{1}^{T} P D_{1}\right) \Theta_{1}^{*}-\left(\Theta_{2}^{*}\right)^{T}\left(R_{22}+D_{2}^{T} P D_{2}\right) \Theta_{2}^{*} \\
& +\left[\left(\Theta_{2}^{*}\right)^{T}\left(R_{22}+D_{2}^{T} P D_{2}\right)+\left(P B_{2}+C^{T} P D_{2}+S_{2}^{T}\right)\right] \Theta_{2}^{*} \\
& +\left(\Theta_{2}^{*}\right)^{T}\left[\left(B_{2}^{T} P+D_{2}^{T} P C+S_{2}\right)+\left(R_{22}+D_{2}^{T} P D_{2}\right) \Theta_{2}^{*}\right] \\
= & P A+A^{T} P+C^{T} P C+Q-\left(\Theta_{1}^{*}\right)^{T}\left(R_{11}+D_{1}^{T} P D_{1}\right) \Theta_{1}^{*}-\left(\Theta_{2}^{*}\right)^{T}\left(R_{22}+D_{2}^{T} P D_{2}\right) \Theta_{2}^{*} \\
& -\left(\Theta_{1}^{*}\right)^{T}\left(D_{1}^{T} P D_{2}+R_{12}\right) \Theta_{2}^{*}-\left(\Theta_{2}^{*}\right)^{T}\left(D_{2}^{T} P D_{1}+R_{21}\right) \Theta_{1}^{*} \\
= & P A+A^{T} P+C^{T} P C+Q-\left(\Theta^{*}\right)^{T}\left(R+D^{T} P D\right) \Theta^{*} \\
= & P A+A^{T} P+C^{T} P C+Q-\left(P B+C^{T} P D+S^{T}\right)\left(R+D^{T} P D\right)^{\dagger}\left(B^{T} P+D^{T} P C+S\right) .
\end{aligned}
$$

Therefore, $P$ is a stabilizing solution of ARE (5.10). 
The following result, which is the main result of this paper, gives a characterization for closed-loop saddle points of Problem (LQG).

Theorem 5.7. Problem (LQG) admits a closed-loop saddle point $\left(\Theta^{*}, u^{*}(\cdot)\right) \in \mathbb{R}^{m \times n} \times L_{\mathbb{F}}^{2}\left(\mathbb{R}^{m}\right)$ with $\Theta^{*} \equiv$ $\left(\left(\Theta_{1}^{*}\right)^{T},\left(\Theta_{2}^{*}\right)^{T}\right)^{T}$ and $u^{*}(\cdot) \equiv\left(u_{1}^{*}(\cdot)^{T}, u_{2}^{*}(\cdot)^{T}\right)^{T}$ if and only if the following hold:

(i) ARE (5.10) admits a stabilizing solution P;

(ii) The following BSDE:

$$
\begin{aligned}
\mathrm{d} \eta= & -\left\{\left[A^{T}-\mathscr{L}(P) \mathscr{N}(P)^{\dagger} B^{T}\right] \eta+\left[C^{T}-\mathscr{L}(P) \mathscr{N}(P)^{\dagger} D^{T}\right] \zeta\right. \\
& \left.+\left[C^{T}-\mathscr{L}(P) \mathscr{N}(P)^{\dagger} D^{T}\right] P \sigma-\mathscr{L}(P) \mathscr{N}(P)^{\dagger} \rho+P b+q\right\} \mathrm{d} t+\zeta \mathrm{d} W(t), \quad t \geq 0
\end{aligned}
$$

admits an $L^{2}$-stable adapted solution $(\eta(\cdot), \zeta(\cdot))$ such that

$$
B^{T} \eta(t)+D^{T} \zeta(t)+D^{T} P \sigma(t)+\rho(t) \in \mathcal{R}(\mathscr{N}(P)), \quad \text { a.e. } t \in[0, \infty) \text {, a.s. }
$$

In this case, the closed-loop saddle point $\left(\Theta^{*}, u^{*}(\cdot)\right)$ admits the following representation:

$$
\left\{\begin{array}{l}
\Theta^{*}=-\mathscr{N}(P)^{\dagger} \mathscr{L}(P)^{T}+\left[I-\mathscr{N}(P)^{\dagger} \mathscr{N}(P)\right] \Pi, \\
u^{*}(\cdot)=-\mathscr{N}(P)^{\dagger}\left[B^{T} \eta(\cdot)+D^{T} \zeta(\cdot)+D^{T} P \sigma(\cdot)+\rho(\cdot)\right]+\left[I-\mathscr{N}(P)^{\dagger} \mathscr{N}(P)\right] \nu(\cdot),
\end{array}\right.
$$

where $\Pi \in \mathbb{R}^{m \times n}$ is chosen such that $\Theta^{*} \in \mathscr{S}[A, C ; B, D]$, and $\nu(\cdot) \in L_{\mathbb{F}}^{2}\left(\mathbb{R}^{m}\right)$.

Further, the value function admits the following representation:

$$
\begin{aligned}
V(x)= & \langle P x, x\rangle+\mathbb{E}\left\{2\langle\eta(0), x\rangle+\int_{0}^{\infty}[\langle P \sigma, \sigma\rangle+2\langle\eta, b\rangle+2\langle\zeta, \sigma\rangle\right. \\
& \left.\left.-\left\langle\left(R+D^{T} P D\right)^{\dagger}\left(B^{T} \eta+D^{T} \zeta+D^{T} P \sigma+\rho\right), B^{T} \eta+D^{T} \zeta+D^{T} P \sigma+\rho\right\rangle\right] \mathrm{d} t\right\} .
\end{aligned}
$$

Proof.

Necessity. Let $\left(\Theta^{*}, u^{*}(\cdot)\right) \in \mathbb{R}^{m \times n} \times L_{\mathbb{F}}^{2}\left(\mathbb{R}^{m}\right)$ be a closed-loop saddle point of Problem (LQG) with $\Theta^{*} \equiv$ $\left(\left(\Theta_{1}^{*}\right)^{T},\left(\Theta_{2}^{*}\right)^{T}\right)^{T}$ and $u^{*}(\cdot) \equiv\left(u_{1}^{*}(\cdot)^{T}, u_{2}^{*}(\cdot)^{T}\right)^{T}$. It follows from Proposition 5.4 that $\left(\Theta_{1}^{*}, 0 ; \Theta_{2}^{*}, 0\right)$ is a closedloop saddle point of Problem (LQG) ${ }^{0}$. By Proposition 5.6, ARE (5.10) admits a stabilizing solution $P$, and $\Theta^{*}$ is given by (5.15).

To determine $u^{*}(\cdot)$, let $\left(X^{*}(\cdot), Y^{*}(\cdot), Z^{*}(\cdot)\right)$ be the solution of (5.6). Then

$$
R u^{*}+B^{T} Y^{*}+D^{T} Z^{*}+\left(S+R \Theta^{*}\right) X^{*}+\rho=0, \quad \text { a.e. a.s. }
$$

and hence,

$$
\begin{aligned}
\mathrm{d} Y^{*}= & -\left\{\left(A+B \Theta^{*}\right)^{T} Y^{*}+\left(C+D \Theta^{*}\right)^{T} Z^{*}+\widetilde{Q} X^{*}+\widetilde{S}^{T} u^{*}+\widetilde{q}\right\} \mathrm{d} t+Z^{*} \mathrm{~d} W(t) \\
= & -\left\{A^{T} Y^{*}+C^{T} Z^{*}+\left(Q+S^{T} \Theta^{*}\right) X^{*}+S^{T} u^{*}+q\right. \\
& \left.+\left(\Theta^{*}\right)^{T}\left[B^{T} Y^{*}+D^{T} Z^{*}+\left(S+R \Theta^{*}\right) X^{*}+R u^{*}+\rho\right]\right\} \mathrm{d} t+Z^{*} \mathrm{~d} W(t) \\
= & -\left\{A^{T} Y^{*}+C^{T} Z^{*}+\left(Q+S^{T} \Theta^{*}\right) X^{*}+S^{T} u^{*}+q\right\} \mathrm{d} t+Z^{*} \mathrm{~d} W(t), \quad t \geq 0 .
\end{aligned}
$$


Define

$$
\left\{\begin{array}{l}
\eta(t)=Y^{*}(t)-P X^{*}(t) \\
\zeta(t)=Z^{*}(t)-P\left(C+D \Theta^{*}\right) X^{*}(t)-P D u^{*}(t)-P \sigma(t), \quad t \geq 0
\end{array}\right.
$$

Noting $\mathscr{M}(P)+\mathscr{L}(P) \Theta^{*}=0$, we have

$$
\begin{aligned}
\mathrm{d} \eta= & \mathrm{d} Y^{*}-P \mathrm{~d} X^{*} \\
= & -\left[A^{T} Y^{*}+C^{T} Z^{*}+\left(Q+S^{T} \Theta^{*}\right) X^{*}+S^{T} u^{*}+q\right] \mathrm{d} t+Z^{*} \mathrm{~d} W \\
& -P\left[\left(A+B \Theta^{*}\right) X^{*}+B u^{*}+b\right] \mathrm{d} t-P\left[\left(C+D \Theta^{*}\right) X^{*}+D u^{*}+\sigma\right] \mathrm{d} W \\
= & -\left\{A^{T}\left(\eta+P X^{*}\right)+C^{T}\left[\zeta+P\left(C+D \Theta^{*}\right) X^{*}+P D u^{*}+P \sigma\right]\right. \\
& \left.+\left(Q+S^{T} \Theta^{*}\right) X^{*}+S^{T} u^{*}+q+P\left[\left(A+B \Theta^{*}\right) X^{*}+B u^{*}+b\right]\right\} \mathrm{d} t+\zeta \mathrm{d} W \\
= & -\left\{A^{T} \eta+C^{T} \zeta+\mathscr{M}(P) X^{*}+\mathscr{L}(P) \Theta^{*} X^{*}+\mathscr{L}(P) u^{*}+C^{T} P \sigma+P b+q\right\} \mathrm{d} t+\zeta \mathrm{d} W \\
= & -\left[A^{T} \eta+C^{T} \zeta+\mathscr{L}(P) u^{*}+C^{T} P \sigma+P b+q\right] \mathrm{d} t+\zeta \mathrm{d} W .
\end{aligned}
$$

According to $(5.21)$, we have (noting $\mathscr{L}(P)^{T}+\mathscr{N}(P) \Theta^{*}=0$ )

$$
\begin{aligned}
0 & =B^{T} Y^{*}+D^{T} Z^{*}+\left(S+R \Theta^{*}\right) X^{*}+R u^{*}+\rho \\
& =B^{T}\left(\eta+P X^{*}\right)+D^{T}\left[\zeta+P\left(C+D \Theta^{*}\right) X^{*}+P D u^{*}+P \sigma\right]+\left(S+R \Theta^{*}\right) X^{*}+R u^{*}+\rho \\
& =\left[\mathscr{L}(P)^{T}+\mathscr{N}(P) \Theta^{*}\right] X^{*}+B^{T} \eta+D^{T} \zeta+D^{T} P \sigma+\rho+\mathscr{N}(P) u^{*} \\
& =B^{T} \eta+D^{T} \zeta+D^{T} P \sigma+\rho+\mathscr{N}(P) u^{*} .
\end{aligned}
$$

Hence,

$$
B^{T} \eta+D^{T} \zeta+D^{T} P \sigma+\rho \in \mathcal{R}(\mathscr{N}(P)), \quad \text { a.e. a.s. }
$$

Since $\mathscr{N}(P)^{\dagger}\left(B^{T} \eta+D^{T} \zeta+D^{T} P \sigma+\rho\right)=-\mathscr{N}(P)^{\dagger} \mathscr{N}(P) u^{*}$, and $\mathscr{N}(P)^{\dagger} \mathscr{N}(P)$ is an orthogonal projection, we have

$$
u^{*}=-\mathscr{N}(P)^{\dagger}\left(B^{T} \eta+D^{T} \zeta+D^{T} P \sigma+\rho\right)+\left[I-\mathscr{N}(P)^{\dagger} \mathscr{N}(P)\right] \nu
$$

for some $\nu(\cdot) \in L_{\mathbb{F}}^{2}\left(\mathbb{R}^{m}\right)$. Consequently,

$$
\begin{aligned}
\mathscr{L}(P) u^{*} & =-\mathscr{L}(P) \mathscr{N}(P)^{\dagger}\left(B^{T} \eta+D^{T} \zeta+D^{T} P \sigma+\rho\right)+\mathscr{L}(P)\left[I-\mathscr{N}(P)^{\dagger} \mathscr{N}(P)\right] \nu \\
& =-\mathscr{L}(P) \mathscr{N}(P)^{\dagger}\left(B^{T} \eta+D^{T} \zeta+D^{T} P \sigma+\rho\right) .
\end{aligned}
$$

Then

$$
\begin{aligned}
& A^{T} \eta+C^{T} \zeta+\mathscr{L}(P) u^{*}+C^{T} P \sigma+P b+q \\
& =A^{T} \eta+C^{T} \zeta-\mathscr{L}(P) \mathscr{N}(P)^{\dagger}\left(B^{T} \eta+D^{T} \zeta+D^{T} P \sigma+\rho\right)+C^{T} P \sigma+P b+q \\
& =\left[A^{T}-\mathscr{L}(P) \mathscr{N}(P)^{\dagger} B^{T}\right] \eta+\left[C^{T}-\mathscr{L}(P) \mathscr{N}(P)^{\dagger} D^{T}\right] \zeta \\
& \quad+\left[C^{T}-\mathscr{L}(P) \mathscr{N}(P)^{\dagger} D^{T}\right] P \sigma-\mathscr{L}(P) \mathscr{N}(P)^{\dagger} \rho+P b+q .
\end{aligned}
$$

Therefore, $(\eta, \zeta)$ is an $L^{2}$-stable solution to (5.17). 
Sufficiency. Let $\left(\Theta^{*}, u^{*}(\cdot)\right)$ be given by (5.19), where $\Pi \in \mathbb{R}^{m \times n}$ is chosen so that $\Theta^{*} \in \mathscr{S}[A, C ; B, D]$. Then

$$
\begin{gathered}
\mathscr{N}(P) \Theta^{*}+\mathscr{L}(P)^{T}=0, \quad \mathscr{M}(P)+\mathscr{L}(P) \Theta^{*}+\left(\Theta^{*}\right)^{T} \mathscr{L}(P)^{T}+\left(\Theta^{*}\right)^{T} \mathscr{N}(P) \Theta^{*}=0, \\
B^{T} \eta+D^{T} \zeta+D^{T} P \sigma+\rho=-\mathscr{N}(P) u^{*},
\end{gathered}
$$

and

$$
\left[\left(\Theta^{*}\right)^{T}+\mathscr{L}(P) \mathscr{N}(P)^{\dagger}\right]\left(B^{T} \eta+D^{T} \zeta+D^{T} P \sigma+\rho\right)=-\Pi^{T}\left[I-\mathscr{N}(P) \mathscr{N}(P)^{\dagger}\right] \mathscr{N}(P) u^{*}=0 .
$$

We take any $u(\cdot)=\left(u_{1}(\cdot)^{T}, u_{2}(\cdot)^{T}\right)^{T} \in L_{\mathbb{F}}^{2}\left(\mathbb{R}^{m_{1}}\right) \times L_{\mathbb{F}}^{2}\left(\mathbb{R}^{m_{2}}\right)$, and let $X(\cdot) \equiv X(\cdot ; x, u(\cdot))$ be the solution of the following closed-loop system:

$$
\left\{\begin{array}{l}
\mathrm{d} X(t)=\left[\left(A+B \Theta^{*}\right) X(t)+B u(t)+b(t)\right] \mathrm{d} t+\left[\left(C+D \Theta^{*}\right) X(t)+D u(t)+\sigma(t)\right] \mathrm{d} W(t), \quad t \geq 0 \\
X(0)=x
\end{array}\right.
$$

Then

$$
\begin{aligned}
& J\left(x ; \Theta^{*} X(\cdot)+u(\cdot)\right)=\mathbb{E} \int_{0}^{\infty}\left[\left\langle\left(\begin{array}{cc}
Q & S^{T} \\
S & R
\end{array}\right)\left(\begin{array}{c}
X \\
\Theta^{*} X+u
\end{array}\right),\left(\begin{array}{c}
X \\
\Theta^{*} X+u
\end{array}\right)\right\rangle+2\left\langle\left(\begin{array}{c}
q \\
\rho
\end{array}\right),\left(\begin{array}{c}
X \\
\Theta^{*} X+u
\end{array}\right)\right\rangle\right] \mathrm{d} t \\
& =\mathbb{E} \int_{0}^{\infty}\left\{\left\langle\left[Q+S^{T} \Theta^{*}+\left(\Theta^{*}\right)^{T} S+\left(\Theta^{*}\right)^{T} R \Theta^{*}\right] X, X\right\rangle+2\left\langle\left(S+R \Theta^{*}\right) X, u\right\rangle\right. \\
& \left.+\langle R u, u\rangle+2\left\langle q+\left(\Theta^{*}\right)^{T} \rho, X\right\rangle+2\langle\rho, u\rangle\right\} \mathrm{d} t .
\end{aligned}
$$

Applying Itô's formula to $t \mapsto\langle P X(t), X(t)\rangle$, one has (noting (5.22))

$$
\begin{aligned}
\langle P x, x\rangle= & -\mathbb{E} \int_{0}^{\infty}\left\{\left\langle\left[P\left(A+B \Theta^{*}\right)+\left(A+B \Theta^{*}\right)^{T} P\right] X, X\right\rangle+\left\langle P\left(C+D \Theta^{*}\right) X,\left(C+D \Theta^{*}\right) X\right\rangle\right. \\
& \left.\left.+2\langle P X, B u+b\rangle+2\left\langle P\left(C+D \Theta^{*}\right) X, D u+\sigma\right\rangle\right)+\langle P(D u+\sigma), D u+\sigma\rangle\right\} \mathrm{d} t \\
= & -\mathbb{E} \int_{0}^{\infty}\left\{\left\langle\left[\left(P A+A^{T} P+C^{T} P C\right)+\left(P B+C^{T} P D\right) \Theta^{*}+\left(\Theta^{*}\right)^{T}\left(B^{T} P+D^{T} P C\right)\right.\right.\right. \\
& \left.\left.+\left(\Theta^{*}\right)^{T} D^{T} P D \Theta^{*}\right] X, X\right\rangle+2\left\langle\left(B^{T} P+D^{T} P C+D^{T} P D \Theta^{*}\right) X, u\right\rangle \\
& \left.+2\left\langle P\left(C+D \Theta^{*}\right) X, \sigma\right\rangle+\left\langle D^{T} P D u, u\right\rangle+2\left\langle D^{T} P \sigma, u\right\rangle+2\langle P X, b\rangle+\langle P \sigma, \sigma\rangle\right\} \mathrm{d} t \\
= & -\mathbb{E} \int_{0}^{\infty}\left\{\left\langle\left[\mathscr{M}(P)+\mathscr{L}(P) \Theta^{*}+\left(\Theta^{*}\right)^{T} \mathscr{L}(P)^{T}+\left(\Theta^{*}\right)^{T} \mathscr{N}(P) \Theta^{*}\right] X, X\right\rangle\right. \\
& -\left\langle\left[Q+S^{T} \Theta^{*}+\left(\Theta^{*}\right)^{T} S+\left(\Theta^{*}\right)^{T} R \Theta^{*}\right] X, X\right\rangle \\
& +2\left\langle\left[\mathscr{L}(P)^{T}+\mathscr{N}(P) \Theta^{*}-\left(S+R \Theta^{*}\right)\right] X, u\right\rangle \\
& \left.+2\left\langle P\left(C+D \Theta^{*}\right) X, \sigma\right\rangle+\left\langle D^{T} P D u, u\right\rangle+2\left\langle D^{T} P \sigma, u\right\rangle+2\langle P X, b\rangle+\langle P \sigma, \sigma\rangle\right\} \mathrm{d} t \\
= & -\mathbb{E} \int_{0}^{\infty}\left[2\left\langle P\left(C+D \Theta^{*}\right) X, \sigma\right\rangle+\left\langle D^{T} P D u, u\right\rangle+2\left\langle D^{T} P \sigma, u\right\rangle+2\langle P X, b\rangle+\langle P \sigma, \sigma\rangle\right] \mathrm{d} t \\
& +\mathbb{E} \int_{0}^{\infty}\left\langle\left[Q+S^{T} \Theta^{*}+\left(\Theta^{*}\right)^{T} S+\left(\Theta^{*}\right)^{T} R \Theta^{*}\right] X, X\right\rangle+2\left\langle\left(S+R \Theta^{*}\right) X, u\right\rangle \mathrm{d} t .
\end{aligned}
$$


Applying Itô's formula to $t \mapsto\langle\eta(t), X(t)\rangle$, one has (noting (5.24))

$$
\begin{aligned}
\mathbb{E}\langle\eta(0), x\rangle= & \mathbb{E} \int_{0}^{\infty}\left\{\left\langle\left[A^{T}-\mathscr{L}(P) \mathscr{N}(P)^{\dagger} B^{T}\right] \eta+\left[C^{T}-\mathscr{L}(P) \mathscr{N}(P)^{\dagger} D^{T}\right] \zeta\right.\right. \\
& \left.+\left[C^{T}-\mathscr{L}(P) \mathscr{N}(P)^{\dagger} D^{T}\right] P \sigma-\mathscr{L}(P) \mathscr{N}(P)^{\dagger} \rho+P b+q, X\right\rangle \\
& \left.-\left\langle\left(A+B \Theta^{*}\right) X+B u+b, \eta\right\rangle-\left\langle\zeta,\left(C+D \Theta^{*}\right) X+D u+\sigma\right\rangle\right\} \mathrm{d} t \\
= & \mathbb{E} \int_{0}^{\infty}\left\{-\left\langle\left[\left(\Theta^{*}\right)^{T}+\mathscr{L}(P) \mathscr{N}(P)^{\dagger}\right] B^{T} \eta+\left[\left(\Theta^{*}\right)^{T}+\mathscr{L}(P) \mathscr{N}(P)^{\dagger}\right] D^{T} \zeta, X\right\rangle\right. \\
& -\left\langle\left[\left(\Theta^{*}\right)^{T}+\mathscr{L}(P) \mathscr{N}(P)^{\dagger}\right] D^{T} P \sigma, X\right\rangle+\left\langle P\left(C+D \Theta^{*}\right) X, \sigma\right\rangle \\
& \left.-\left\langle\mathscr{L}(P) \mathscr{N}(P)^{\dagger} \rho, X\right\rangle+\langle P b+q, X\rangle-\langle B u+b, \eta\rangle-\langle\zeta, D u+\sigma\rangle\right\} \mathrm{d} t \\
= & \mathbb{E} \int_{0}^{\infty}\left\{-\left\langle\left[\left(\Theta^{*}\right)^{T}+\mathscr{L}(P) \mathscr{N}(P)^{\dagger}\right]\left(B^{T} \eta+D^{T} \zeta+D^{T} P \sigma+\rho\right), X\right\rangle\right. \\
& \left.+\left\langle P\left(C+D \Theta^{*}\right) X, \sigma\right\rangle+\left\langle\left(\Theta^{*}\right)^{T} \rho+P b+q, X\right\rangle-\langle B u+b, \eta\rangle-\langle\zeta, D u+\sigma\rangle\right\} \mathrm{d} t \\
= & \mathbb{E} \int_{0}^{\infty}\left\{\left\langle P\left(C+D \Theta^{*}\right) X, \sigma\right\rangle+\left\langle\left(\Theta^{*}\right)^{T} \rho+P b+q, X\right\rangle-\langle B u+b, \eta\rangle-\langle\zeta, D u+\sigma\rangle\right\} \mathrm{d} t .
\end{aligned}
$$

Combining (5.25)-(5.27) and noting (5.23), we have

$$
\begin{aligned}
& J\left(x ; \Theta^{*} X(\cdot)+u(\cdot)\right)-\langle P x, x\rangle-2 \mathbb{E}\langle\eta(0), x\rangle \\
& =\mathbb{E} \int_{0}^{\infty}\left\{\langle\mathscr{N}(P) u, u\rangle+2\left\langle B^{T} \eta+D^{T} \zeta+D^{T} P \sigma+\rho, u\right\rangle+2\langle b, \eta\rangle+2\langle\zeta, \sigma\rangle+\langle P \sigma, \sigma\rangle\right\} \mathrm{d} t \\
& =\mathbb{E} \int_{0}^{\infty}\left\{\langle\mathscr{N}(P) u, u\rangle-2\left\langle\mathscr{N}(P) u^{*}, u\right\rangle+2\langle b, \eta\rangle+2\langle\zeta, \sigma\rangle+\langle P \sigma, \sigma\rangle\right\} \mathrm{d} t \\
& =\mathbb{E} \int_{0}^{\infty}\left\{\left\langle\mathscr{N}(P)\left(u-u^{*}\right), u-u^{*}\right\rangle-\left\langle\mathscr{N}(P) u^{*}, u^{*}\right\rangle+2\langle b, \eta\rangle+2\langle\zeta, \sigma\rangle+\langle P \sigma, \sigma\rangle\right\} \mathrm{d} t .
\end{aligned}
$$

Consequently,

$$
\begin{aligned}
& J\left(x ; \Theta_{1}^{*} X(\cdot)+u_{1}(\cdot), \Theta_{2}^{*} X(\cdot)+u_{2}^{*}(\cdot)\right)-J\left(x ; \Theta^{*} X^{*}(\cdot)+u^{*}(\cdot)\right) \\
& =\mathbb{E} \int_{0}^{\infty}\left\langle\left(R_{11}+D_{1}^{T} P D_{1}\right)\left(u_{1}-u_{1}^{*}\right), u_{1}-u_{1}^{*}\right\rangle \mathrm{d} t \geq 0
\end{aligned}
$$

since $R_{11}+D_{1}^{T} P D_{1} \geq 0$. Similarly,

$$
\begin{aligned}
& J\left(x ; \Theta_{1}^{*} X(\cdot)+u_{1}^{*}(\cdot), \Theta_{2}^{*} X(\cdot)+u_{2}(\cdot)\right)-J\left(x ; \Theta^{*} X^{*}(\cdot)+u^{*}(\cdot)\right) \\
& =\mathbb{E} \int_{0}^{\infty}\left\langle\left(R_{22}+D_{2}^{T} P D_{2}\right)\left(u_{2}-u_{2}^{*}\right), u_{2}-u_{2}^{*}\right\rangle \mathrm{d} t \leq 0
\end{aligned}
$$

since $R_{22}+D_{2}^{T} P D_{2} \leq 0$. Therefore, $\left(\Theta^{*}, u^{*}(\cdot)\right)$ is a closed-loop saddle point of Problem (LQG). Finally, noting (5.23), we have

$$
\begin{aligned}
\left\langle\mathscr{N}(P) u^{*}, u^{*}\right\rangle & =\left\langle\mathscr{N}(P) \mathscr{N}(P)^{\dagger} \mathscr{N}(P) u^{*}, u^{*}\right\rangle=\left\langle\mathscr{N}(P)^{\dagger} \mathscr{N}(P) u^{*}, \mathscr{N}(P) u^{*}\right\rangle \\
& =\left\langle\left(R+D^{T} P D\right)^{\dagger}\left(B^{T} \eta+D^{T} \zeta+D^{T} P \sigma+\rho\right), B^{T} \eta+D^{T} \zeta+D^{T} P \sigma+\rho\right\rangle,
\end{aligned}
$$


and hence,

$$
\begin{aligned}
V(x)= & J\left(x ; \Theta^{*} X(\cdot)+u^{*}(\cdot)\right) \\
= & \langle P x, x\rangle+2 \mathbb{E}\langle\eta(0), x\rangle+\mathbb{E} \int_{0}^{\infty}\left\{-\left\langle\mathscr{N}(P) u^{*}, u^{*}\right\rangle+2\langle b, \eta\rangle+2\langle\zeta, \sigma\rangle+\langle P \sigma, \sigma\rangle\right\} \mathrm{d} t \\
= & \langle P x, x\rangle+\mathbb{E}\left\{2\langle\eta(0), x\rangle+\int_{0}^{\infty}[\langle P \sigma, \sigma\rangle+2\langle\eta, b\rangle+2\langle\zeta, \sigma\rangle\right. \\
& \left.\left.-\left\langle\left(R+D^{T} P D\right)^{\dagger}\left(B^{T} \eta+D^{T} \zeta+D^{T} P \sigma+\rho\right), B^{T} \eta+D^{T} \zeta+D^{T} P \sigma+\rho\right\rangle\right] \mathrm{d} t\right\} .
\end{aligned}
$$

This completes the proof.

Note that the above result is reduced to that for Problem (LQ) if $m_{2}=0$. It is not hard for us to state such a result and we omit the details here.

\section{EXAMPLES}

In this section we present some examples illustrating how the "stabilizing solution" of AREs plays an important role in the study of closed-loop saddle points.

The first example shows that the algebraic Riccati equation may only admits non-stabilizing solutions even if the system $[A, C ; B, D]$ is stabilizable.

Example 6.1. Consider the following one-dimensional state equation

$$
\left\{\begin{array}{l}
\mathrm{d} X(t)=-\frac{1}{2} X(t) \mathrm{d} t+\left[u_{1}(t)+u_{2}(t)\right] \mathrm{d} W(t), \quad t \geq 0, \\
X(0)=x,
\end{array}\right.
$$

with the performance functional

$$
J\left(x ; u_{1}(\cdot), u_{2}(\cdot)\right)=\mathbb{E} \int_{0}^{\infty}\left\langle\left(\begin{array}{ccc}
1 & 1 & -1 \\
1 & 1 & 0 \\
-1 & 0 & -1
\end{array}\right)\left(\begin{array}{l}
X(t) \\
u_{1}(t) \\
u_{2}(t)
\end{array}\right),\left(\begin{array}{l}
X(t) \\
u_{1}(t) \\
u_{2}(t)
\end{array}\right)\right\rangle \mathrm{d} t .
$$

In this example,

$$
\left\{\begin{array}{l}
A=-\frac{1}{2}, \quad B=(0,0), \quad C=0, \quad D=(1,1), \\
Q=1, \quad S=(1,-1)^{T}, \quad R=\left(\begin{array}{cc}
1 & 0 \\
0 & -1
\end{array}\right) .
\end{array}\right.
$$

By Lemma 2.3, part (iv), the system $[A, C ; B, D]$ is stabilizable, and $\Theta=\left(\Theta_{1}, \Theta_{2}\right)^{T} \in \mathscr{S}[A, C ; B, D]$ if and only if

$$
\left.-1+\left(\Theta_{1}+\Theta_{2}\right)^{2}=2(A+B \Theta)+(C+D \Theta)^{2}<0 \quad \text { (i.e., }-1<\Theta_{1}+\Theta_{2}<1\right) .
$$

Note that $R+D^{T} P D$ is invertible for all $P \in \mathbb{R}$ with

$$
\left(R+D^{T} P D\right)^{-1}=\left(\begin{array}{cc}
P+1 & P \\
P & P-1
\end{array}\right)^{-1}=\left(\begin{array}{cc}
-P+1 & P \\
P & -P-1
\end{array}\right) .
$$

Then the corresponding ARE reads

$$
\begin{aligned}
0 & =P A+A^{T} P+C^{T} P C+Q-\left(P B+C^{T} P D+S^{T}\right)\left(R+D^{T} P D\right)^{\dagger}\left(B^{T} P+D^{T} P C+S\right) \\
& =-P+1-(1,-1)\left(\begin{array}{cc}
-P+1 & P \\
P & -P-1
\end{array}\right)\left(\begin{array}{c}
1 \\
-1
\end{array}\right) \\
& =3 P+1 .
\end{aligned}
$$


Thus, $P=-1 / 3$ and

$$
R_{11}+D_{1}^{T} P D_{1}=\frac{2}{3} \geq 0, \quad R_{22}+D_{2}^{T} P D_{2}=-\frac{4}{3} \leq 0 .
$$

Also, the range condition

$$
\mathcal{R}\left(B^{T} P+D^{T} P C+S\right) \subseteq \mathcal{R}\left(R+D^{T} P D\right)
$$

holds automatically since $R+D^{T} P D$ is invertible. However, we have

$$
\left[I-\mathscr{N}(P)^{\dagger} \mathscr{N}(P)\right] \Pi-\mathscr{N}(P)^{\dagger} \mathscr{L}(P)^{T} \equiv\left(-\frac{5}{3},-\frac{1}{3}\right)^{T}, \quad \forall \Pi \in \mathbb{R},
$$

which is not a stabilizer of the system $[A, C ; B, D]$. Hence, by Theorem 5.7 , the above problem does not admit closed-loop saddle points. From this example, we see that ARE (5.10) may only admit non-stabilizing solutions.

Next we give an example of Problem $(\mathrm{LQG})^{0}$ which admits uncountably many closed-loop saddle points. It also tells us when the algebraic Riccati equation is solvable, $-\mathscr{N}(P)^{\dagger} \mathscr{L}(P)^{T}$ may not be a stabilizer of the system $[A, C ; B, D]$ in general, and we should carefully choose $\Pi$ so that $\left[I-\mathscr{N}(P)^{\dagger} \mathscr{N}(P)\right] \Pi-\mathscr{N}(P)^{\dagger} \mathscr{L}(P)^{T}$ is a closed-loop saddle point of the game.

Example 6.2. Consider the following one-dimensional state equation

$$
\left\{\begin{array}{l}
\mathrm{d} X(t)=-\left[\frac{1}{4} X(t)+\frac{1}{2} u_{2}(t)\right] \mathrm{d} t+\left[-X(t)+u_{1}(t)\right] \mathrm{d} W(t), \quad t \geq 0, \\
X(0)=x
\end{array}\right.
$$

with the performance functional

$$
J\left(x ; u_{1}(\cdot), u_{2}(\cdot)\right)=\mathbb{E} \int_{0}^{\infty}\left\langle\left(\begin{array}{ccc}
\frac{1}{2} & -1 & -\frac{1}{2} \\
-1 & 1 & 0 \\
-\frac{1}{2} & 0 & 0
\end{array}\right)\left(\begin{array}{l}
X(t) \\
u_{1}(t) \\
u_{2}(t)
\end{array}\right),\left(\begin{array}{l}
X(t) \\
u_{1}(t) \\
u_{2}(t)
\end{array}\right)\right\rangle \mathrm{d} t .
$$

In this example,

$$
\left\{\begin{array}{l}
A=-\frac{1}{4}, \quad B=\left(0,-\frac{1}{2}\right), \quad C=-1, \quad D=(1,0), \\
Q=\frac{1}{2}, \quad S=\left(-1,-\frac{1}{2}\right)^{T}, \quad R=\left(\begin{array}{ll}
1 & 0 \\
0 & 0
\end{array}\right) .
\end{array}\right.
$$

By Lemma 2.3, part (iv), $\Theta=\left(\Theta_{1}, \Theta_{2}\right)^{T} \in \mathscr{S}[A, C ; B, D]$ if and only if

$$
2\left(-\frac{1}{4}-\frac{1}{2} \Theta_{2}\right)+\left(-1+\Theta_{1}\right)^{2}=2(A+B \Theta)+(C+D \Theta)^{2}<0
$$

that is,

$$
\Theta_{1}^{2}-2 \Theta_{1}+\frac{1}{2}<\Theta_{2}
$$

The corresponding ARE reads

$$
\begin{aligned}
0 & =P A+A^{T} P+C^{T} P C+Q-\left(P B+C^{T} P D+S^{T}\right)\left(R+D^{T} P D\right)^{\dagger}\left(B^{T} P+D^{T} P C+S\right) \\
& =\frac{1}{2}(P+1)-\left(-(P+1),-\frac{1}{2}(P+1)\right)\left(\begin{array}{rr}
P+1 & 0 \\
0 & 0
\end{array}\right)^{\dagger}\left(\begin{array}{c}
-(P+1) \\
-\frac{1}{2}(P+1)
\end{array}\right) \\
& =\frac{1}{2}(P+1)-\frac{1}{4}(P+1)^{2}(2,1)\left(\begin{array}{rr}
P+1 & 0 \\
0 & 0
\end{array}\right)^{\dagger}\left(\begin{array}{l}
2 \\
1
\end{array}\right) .
\end{aligned}
$$


It is easy to verify that $P=-1$ is the unique solution of (6.11). Thus,

$$
\mathscr{N}(P)=\left(R+D^{T} P D\right)=\left(\begin{array}{ll}
0 & 0 \\
0 & 0
\end{array}\right), \quad \mathscr{L}(P)=P B+C^{T} P D+S^{T}=(0,0) .
$$

Hence, all the conditions

$$
R_{11}+D_{1}^{T} P D_{1} \geq 0, \quad R_{22}+D_{2}^{T} P D_{2} \leq 0, \quad \mathcal{R}\left(B^{T} P+D^{T} P C+S\right) \subseteq \mathcal{R}\left(R+D^{T} P D\right)
$$

hold. By Theorem 5.7, we see that

$$
\left(\Theta_{1}, \nu_{1}(\cdot) ; \Theta_{2}, \nu_{2}(\cdot)\right) \quad \text { with } \quad \Theta_{1}^{2}-2 \Theta_{1}+\frac{1}{2}<\Theta_{2}, \quad \nu_{1}(\cdot), \nu_{2}(\cdot) \in L_{\mathbb{F}}^{2}(\mathbb{R})
$$

are all the closed-loop saddle points of the above problem. However,

$$
-\mathscr{N}(P)^{\dagger} \mathscr{L}(P)^{T}=(0,0)^{T} \notin \mathscr{S}[A, C ; B, D] .
$$

Also, from this example, we see that even if $-\mathscr{N}(P)^{\dagger} \mathscr{L}(P)^{T}$ is not a stabilizer of the system, Problem (LQG) may still admit closed-loop saddle points, thanks to the fact that we can properly choose $\Pi \neq 0$ so that the term $\left[I-\mathscr{N}(P)^{\dagger} \mathscr{N}(P)\right] \Pi$ could play a role.

Finally, we present an example showing that not all of the stabilizers are necessarily closed-loop saddle points of the game. It may happens that the system $[A, C ; B, D]$ have more than one (uncountably many) stabilizer, while the closed-loop saddle point is unique.

Example 6.3. Consider the following one-dimensional state equation

$$
\left\{\begin{array}{l}
\mathrm{d} X(t)=\left[-8 X(t)+u_{1}(t)-u_{2}(t)\right] \mathrm{d} t+\left[u_{1}(t)+u_{2}(t)\right] \mathrm{d} W(t), \quad t \geq 0 \\
X(0)=x
\end{array}\right.
$$

with the performance functional

$$
J\left(x ; u_{1}(\cdot), u_{2}(\cdot)\right)=\mathbb{E} \int_{0}^{\infty}\left[12 X(t)^{2}+u_{1}(t)^{2}-u_{2}(t)^{2}\right] \mathrm{d} t .
$$

In this example,

$$
\begin{cases}A=-8, & B=(1,-1), \quad C=0, \quad D=(1,1), \\
Q=12, & S=(0,0)^{T}, \quad R=\left(\begin{array}{cc}
1 & 0 \\
0 & -1
\end{array}\right) .\end{cases}
$$

By Lemma 2.3, part (iv), $\Theta=\left(\Theta_{1}, \Theta_{2}\right)^{T} \in \mathscr{S}[A, C ; B, D]$ if and only if

$$
-16+2\left(\Theta_{1}-\Theta_{2}\right)+\left(\Theta_{1}+\Theta_{2}\right)^{2}<0 .
$$

The corresponding ARE reads

$$
\begin{aligned}
0 & =P A+A^{T} P+C^{T} P C+Q-\left(P B+C^{T} P D+S^{T}\right)\left(R+D^{T} P D\right)^{\dagger}\left(B^{T} P+D^{T} P C+S\right) \\
& =-16 P+12-P^{2}(1,-1)\left(\begin{array}{cc}
P+1 & P \\
P & P-1
\end{array}\right)^{\dagger}\left(\begin{array}{c}
1 \\
-1
\end{array}\right) \\
& =-16 P+12-P^{2}(1,-1)\left(\begin{array}{cc}
-P+1 & P \\
P & -P-1
\end{array}\right)\left(\begin{array}{c}
1 \\
-1
\end{array}\right) \\
& =4 P^{3}-16 P+12,
\end{aligned}
$$


which has three solutions:

$$
P_{1}=1, \quad P_{2}=\frac{-1+\sqrt{13}}{2}, \quad P_{3}=\frac{-1-\sqrt{13}}{2} .
$$

All of them satisfy the range condition

$$
\mathcal{R}\left(B^{T} P+D^{T} P C+S\right) \subseteq \mathcal{R}\left(R+D^{T} P D\right)
$$

since $R+D^{T} P D$ is invertible for any $P \in \mathbb{R}$. However, only $P_{1}=1$ satisfies

$$
R_{11}+D_{1}^{T} P D_{1} \geq 0, \quad R_{22}+D_{2}^{T} P D_{2} \leq 0 .
$$

For any $\Pi \in \mathbb{R}$,

$$
\left[I-\mathscr{N}\left(P_{1}\right)^{\dagger} \mathscr{N}\left(P_{1}\right)\right] \Pi-\mathscr{N}\left(P_{1}\right)^{\dagger} \mathscr{L}\left(P_{1}\right)^{T} \equiv(1,-3)^{T},
$$

which satisfies (6.15) and hence is a stabilizer of the system $[A, C ; B, D]$. By Theorem 5.7, the above problem admits a unique closed-loop saddle point $(1,0 ;-3,0)$.

On the other hand, by verifying (6.15), we see that

$$
\begin{array}{ll}
\left(\Theta_{1}^{*}, \Theta_{2}^{*}\right)=(1,-3), & \left(\widetilde{\Theta}_{1}, \widetilde{\Theta}_{2}\right)=(0,0), \\
\left(\Theta_{1}^{*}, \widetilde{\Theta}_{2}\right)=(1,0), & \left(\widetilde{\Theta}_{1}, \Theta_{2}^{*}\right)=(0,-3)
\end{array}
$$

are stabilizers of $[A, C ; B, D]$, but only $\left(\Theta_{1}^{*}, \Theta_{2}^{*}\right)$ is the closed-loop saddle point of the problem.

Acknowledgements. The authors would like to thank the anonymous referee for his/her suggestive comments, which leads to an improvement of the paper.

\section{REFERENCES}

[1] M. Ait Rami, X.Y. Zhou and J.B. Moore, Well-posedness and attainability of indefnite stochastic linear quadratic control in infnite time horizon. Syst. Control Lett. 41 (2000) 123-133.

[2] A. Albert, Conditions for positive and nonnegative definiteness in terms of pseudo-inverses. SIAM J. Appl. Math. 17 (1969) 434-440.

[3] T. Basar and P. Bernhard, $H^{\infty}$-Optimal Control and Related Minimax Design Problems: A Dynamic Game Approach, Birkhäuser, Boston (1991) (2nd edn., 1995).

[4] L.D. Berkovitz, Lectures on differential games, Differential Games and Related Topics, edited by H.W. Kuhn and G.P. Szego. North-Holland, Amsterdam, The Netherlands (1971) 3-45.

[5] P. Bernhard, Linear-quadratic, two-person, zero-sum differential games: Necessary and sufficient conditions. J. Optim. Theory Appl. 27 (1979) 51-69.

[6] M.C. Delfour, Linear quadratic differential games: saddle point and Riccati differential equations. SIAM J. Control Optim. 46 (2007) 750-774.

[7] M.C. Delfour and O.D. Sbarba, Linear quadratic differential games: closed loop saddle points. SIAM J. Control Optim. 47 (2009) 3138-3166.

[8] Y.C. Ho, A.E. Bryson and S. Baron, Differential games and optimal pursuit-evasion strategies. IEEE Trans. Automat. Control 10 (1965) 385-389.

[9] J. Huang, X. Li and J. Yong, A linear-quadratic optimal control problem for mean-field stochastic differential equations in infinite horizon. Appl. Math. Optim. 70 (2014) 29-59.

[10] A. Ichikawa, Linear quadratic differential games in a Hilbert space. SIAM J. Control Optim. 14 (1976) 120-136.

[11] I. Karatzas and S.E. Shreve, Brownian Motion and Stochastic Calculus, 2nd edition. Springer-Verlag, New York (1991).

[12] L. Mou, J. Yong, Two-person zero-sum linear quadratic stochastic differential games by a Hilbert space method. J. Ind. Manag. Optim. 2 (2006) 95-117.

[13] S. Peng and Y. Shi, Infinite horizon forward-backward stochastic differential equations. Stochastic Process. Appl. 85 (2000) 75-92.

[14] R. Penrose, A generalized inverse of matrices. Proc. Cambridge Philos. Soc. 52 (1955) 17-19.

[15] W. E. Schmitendorf, Existence of optimal open-loop strategies for a class of differential games. J. Optim. Theory Appl. 5 (1970) 363-375. 
[16] J. Sun and J. Yong, Linear Quadratic Stochastic Differential Games: Open-Loop and Closed-Loop Saddle Points. SIAM J. Control Optim. 52 (2014) 4082-4121.

[17] H. Wu and X.Y. Zhou, Stochastic frequency characteristic. SIAM J. Control Optim. 40 (2001) 557-576.

[18] J. Yong, Linear-Quadratic Optimal Control Problems for Mean-Field Stochastic Differential Equations. SIAM J. Control Optim. 51 (2013) 2809-2838.

[19] J. Yong and X.Y. Zhou, Stochastic Controls: Hamiltonian Systems and HJB Equations. Springer-Verlag, New York (1999).

[20] P. Zhang, Some Results on Two-Person Zero-Sum linear Quadratic Differential Games. SIAM J. Control Optim. 43 (2005) $2157-2165$. 